\title{
Automatic Velocity Analysis via Shot Profile
}

\author{
Migration \\ Peng Shen ${ }^{1}$, William W. Symes ${ }^{2}$ \\ ${ }^{1}$ Shell Internatioal Exploration and Production \\ ${ }^{2}$ Department of Computational and Applied Mathematics, Rice University \\ February 10, 2008

\footnotetext{
${ }^{1}$ Email: peng.shen@shell.com

2 Email: symes@caam.rice.edu
} 


\begin{abstract}
Shot profile migration provides a convenient framework for implementation of a differential semblance algorithm for estimation of complex, strongly refracting velocity fields. The objective function minimized in this algorithm may measure either focussing of the image in offset or flatness of the image in (scattering) angle. Velocity estimation based on this measure of data-model consistency uses waveform data directly: it does not require any sort of traveltime picking. We show that the offset variant of differential semblance yields somewhat more reliable migration velocity estimates than does the scattering angle variant, and explain why this is so. We observe that inconsistency with the underlying model (Born scattering about a transparent background) may lead to degraded velocity estimates from differential semblance, and show how to augment the objective function with stack power to enhance ultimate accuracy. A 2D marine survey over a target obscured by the lensing effects of a gas chimney provides an opportunity for direct comparison of differential semblance with reflection tomography. The differential semblance estimate yields a more data-consistent model (flatter angle gathers) than does reflection tomography in this application, resulting in a more interpretable image below the gas cloud.
\end{abstract}

\title{
Introduction
}

Differential semblance velocity analysis ("DSVA", (Symes, 1986)) estimates prestack migration velocity models directly from waveform data, by means of an automatic numerical optimization process driven by prestack migration and related computations. 
DSVA avoids the data reduction step (event picking) inherent in reflection traveltime tomography in any of its guises. While the reduction (picking) process is largely automated in current practice, manual intervention is sometimes necessary, and in any case tomographic estimates depend intrinsically on the events selected for kinematic interpretation. In contrast, differential semblance uses all events present in (preprocessed) data to drive velocity estimation.

Several authors have presented DSVA methods for laterally heterogeneous velocity based on various forms of prestack migration (Symes and Versteeg, 1993; Kern and Symes, 1994; Chauris and Noble, 2001; Mulder and ten Kroode, 2002b; Foss et al., 2004). Wave equation migration, in either its shot-geophone (survey-sinking) or shotprofile forms, has the very significant advantage in this context of freedom from kinematic artifacts, which makes it especially suitable for velocity analysis in the presence of strong refraction (Stolk and Symes, 2004; Stolk et al., 2005).Shen et al. (Shen et al., 2003) presented a version DSVA based on the double-square-root ("DSR") migration to create an image volume. In this approach, the failure of the migration to focus the image in (subsurface) offset is an index of velocity error. The mean square of the image volume scaled by offset is an objective measure of focusing failure. DSVA uses numerical minimization of this objective to update the velocity iteratively to minimize this objective, and thus enhance the kinematic consistency between data and model.

This paper presents an algorithm similar to that described by Shen et al. (Shen et al., 2003), using shot profile (rather than DSR) migration by depth extrapolation. The main computation needed in DSVA, not provided by typical implementations of shot profile migration, is the computation of the objective gradient. We show how the 
gradient may be computed by adding appropriate computations to the depth stepping loop (this is an example of the adjoint state method).

The assumptions underlying DSVA are identical to those underlying prestack migration itself, and to a considerable extent reflection tomography:

- the wave propagation model exhibits a scale dichotomy, being divided into a smooth or transparent background velocity and a wavelength-scale reflectivity;

- the reflectivity acts as a velocity perturbation about the transparent background (Born approximation).

We also assume for the purposes of this discussion a constant-density acoustic model of wave propagation in the Earth, though this assumption is probably less essential than the other two.

Data predicted from such a model consists entirely of primary reflections. Granted reasonable fidelity to the assumptions outlined in the preceding paragraph, DSVA is able to recover a kinematically accurate velocity estimate in a few tens of iterations, even with strong lateral velocity variation and its attendant focusing and multipathing. We illustrate this capability using a 2D synthetic example based on the Marmousi velocity model (Versteeg, 1993). We create Born data using the high spatial frequencies of Marmousi as perturbation about a smoothed reference model. DSVA is able to adjust the velocity to produce a quite precise image of the reflector structure. Using this example, we compare two possible variants of the objective minimized by DSVA: the offset-domain objective, described above, and an analogous objective that measures flatness of image gathers parametrized by scattering angle (Sava and Fomel, 2003). 
We find that the straightforward measure of flatness, the angle derivative, is more difficult to optimize sucessfully than is the offset domain objective. This difficulty may be understood in terms of the mathematical properties of the two objectives.

Since the assumptions outlined above are quite restrictive, it is important to understand the extent to which they can be relaxed. We use this shot-profile DSVA implementation described below to explore the behaviour of the algorithm with "imperfect" data. First, we apply our shot-profile algorithm to full waveform data generated from the original Marmousi model, without Born approximation. The multiple reflection content of this data is minimal, so the second assumption is still roughly honored (for the effect of strong multiples on DSVA and some partial fixes, see ( $\mathrm{Li}$ and Symes, 2007)). However the (unsmoothed) Marmousi velocity model does not exhibit the scale dichotomy presupposed by the DSVA theory, and the algorithm is considerably less successful. It is simply unable to estimate the small-scale features which determine the fine kinematics of this data. However it does produce a reasonable crude approximation, and this illustrates another feature of DSVA, namely robust initial convergence from more-or-less arbitrary initial models. To refine the ultimate accuracy of the algorithm, we modify DSVA by adding another term to the objective function, measuring stack power. The modified algorithm uses differential semblance to drive the velocity model to the vicinity of a kinematically correct one, then stack power to refine the final model for maximal accuracy. Stack power does not generally converge from inaccurate initial models, but its local convergence from a good initial model appears not to be so dependent on scale-separation as is DSVA (Soubaras and Gratacos, 2007). This idea has been used also by Chauris and Noble (Chauris and Noble, 2001). We find that 
the modified algorithm is able to construct a quite accurate velocity model for prestack migration of the full waveform Marmousi data, starting with a quite inaccurate initial guess.

Second, we apply the modified DSVA method to a 2D marine line, in which the deeper reflectors in one portion of the section are strongly distorted by the presence of a gas chimney. This example affords an opportunity to compare the waveform-driven modified DSVA with reflection tomography. A velocity model obtained by reflection tomography removes much of the pullup evident in the image using an initial $v(z)$ model, but leaves the deeper events poorly imaged beneath the gas. Modified DSVA produces more continuous events beneath the chimney, and moreover considerably improves the scattering angle gathers, showing that the modified DSVA model is more kinematically consistent with the data than is the reflection tomography model. This example, similar to one reported recently by Kabir et al. (Kabir et al., 2007), lends some credence to the concept expressed at the beginning of this introduction, that is, that the use of full waveform data may more effectively constrain the velocity model than does the inevitably sparse selection of events picked for fitting in reflection tomography.

\section{Differential Semblance}

We will discuss explicitly the migration of 2D images, but note that much of the discussion carries over without modification to 3D migration. Image (mid)point coordinates are $x$ and $z$; (migrated) offset, denoted by $h$, is half of the correlation distance between the downward continued source and receiver wavefields. The image volume produced 
by shot-geophone migration will be denoted by $I(x, z, h)$.

We restrict $h$ to be horizontal, as is appropriate when rays carrying significant energy always make an acute angle with the vertical direction (the "DSR assumption"). We also assume that the data is kinematically complete, i.e. that event slownesses determine raypaths uniquely. This is the case for full 3D (areal) acquisition, also for narrow azimuth acquisition provided that crossline structural heterogeneity is mild. Under these assumptions, shot-geophone migration using a kinematically correct velocity focusses the prestack common image at the origin in offset (Stolk et al., 2005). An objective measure of focussing in offset is

$$
J_{h}=\frac{1}{2}\left\|P_{h} I\right\|^{2}=\frac{1}{2} \int h^{2} I^{2}(x, z, h) d x d z d h .
$$

The differential semblance operator $P_{h}=h$ is a zero order differential operator, meaning that it does not change the wavenumber spectrum of $I$. An alternative objective function can be posed to measure the flatness of the image in angle.

$$
J_{\theta}=\frac{1}{2}\left\|P_{\theta} I\right\|^{2}=\frac{1}{2}\left\|\frac{\partial}{\partial \theta} \mathscr{R} I\right\|^{2}
$$

where $\mathscr{R}$ is the Radon transform (Sava and Fomel, 2003) from offset to angle $\theta, \mathscr{R}^{-1}$ its inverse. $J_{\theta}$ also vanishes when the velocity is kinematically correct, under the standing assumptions (Stolk et al., 2005).

\section{Shot Profile Algorithm}

Introduce source $S$ and receiver $R$ wavefields, 


$$
\begin{aligned}
S(x, z, s, \omega) & =\overline{G^{+}(x, z, s, \omega)} \\
R(x, z, s, \omega) & =\int \overline{G^{+}(x, z, r, \omega)} d(r, s, \omega) d r
\end{aligned}
$$

which satisfy the one-way wave equations

$$
\begin{array}{r}
\left(\frac{\partial}{\partial z}-i \sqrt{\frac{\omega^{2}}{c^{2}}+\frac{\partial^{2}}{\partial x^{2}}}\right) S(x, z, s, \omega)=\delta(x-s) \delta(z) \\
\left(\frac{\partial}{\partial z}-i \sqrt{\frac{\omega^{2}}{c^{2}}+\frac{\partial^{2}}{\partial x^{2}}}\right) R(x, z, s, \omega)=\delta(x-r) \delta(z) d(r, s, \omega)
\end{array}
$$

Choose a depth step $\Delta z$, set $z_{k}=k \Delta z, k=0,1,2, \ldots$. Denote by $H\left(c^{k}\right)$ an (approximate) propagator for the operator $\left(\frac{\partial}{\partial z}-i \sqrt{\frac{\omega^{2}}{\left(c^{k}\right)^{2}}+\frac{\partial^{2}}{\partial x^{2}}}\right)$ from $z_{k}$ to $z_{k+1}$, with $c^{k}(x)=c(x, k \Delta z)$. Setting $S^{k}(x, s, \omega)=S(x, k \Delta z, s, \omega)$ and similarly for $R$ we can write the depth extrapolation scheme as

$$
H\left(c^{k}\right) S^{k}=S^{k+1}, H\left(c^{k}\right) R^{k}=R^{k+1}, k=0,1,2, \ldots N_{z}-1
$$

Initial data at the surface is $S^{0}(x, s, \omega)=\delta(x-s)$ and $R^{0}(x, s, \omega)=\int d r \delta(x-$ $r) d(r, s, \omega)$, respectively. Here $H\left(c^{k}\right)$ is a linear operator on the wavefields to be extrapolated from $z=k \Delta z$ to $z=(k+1) \Delta z$. The superscript is used as the depth index for $c, c^{k}=c(\cdot, k \Delta z)$, and the downward continued wavefields $S, R$ and the image in offset $I$ as well. We write the image in offset and depth as

$$
I^{k}(x, h)=\operatorname{Re} \sum_{s, \omega} S^{k}(x-h, s, \omega) R^{k}(x+h, s, \omega)
$$

For either version of $J$, the gradient is

$$
\nabla_{c} J=\left(\frac{\partial I}{\partial c}\right)^{*} P^{*} P I
$$

in which $P=P_{h}$ or $=P_{\theta}$. For convenience, we defined the image residual $D I=$ $P^{*} P I$. 
A recursive computation of the adjoint derivative $(\partial I / \partial c)^{*}$ is also possible; this trick is called the "adjoint state method" in the control literature, and in fact all wave equation migration methods can be viewed as instances (Stolk et al., 2005). See Shen et al. (Shen et al., 2003) for a similar adjoint state computation for DSVA based on DSR migration.

Introduce adjoint state variables $D S, D R$ and $D c$. These fields are related to the input residual field $D I$ by the adjoint state evolution equations

$$
\begin{gathered}
\left(\begin{array}{c}
D S^{k+1} \\
D R^{k+1}
\end{array}\right)=\left(\begin{array}{l}
H\left(c^{k}\right)^{*} D S^{k+1}+\left(A_{S}^{k+1}\right)^{*} D I^{k+1} \\
H\left(c^{k}\right)^{*} D R^{k+1}+\left(A_{R}^{k+1}\right)^{*} D I^{k+1}
\end{array}\right) \\
D c^{k}=\left(\frac{\partial H}{\partial c^{k}} S^{k}\right)^{*} D S^{k+1}+\left(\frac{\partial H}{\partial c^{k}} R^{k}\right)^{*} D R^{k+1} \\
\left(A_{S}^{k}\right) f(x, h, s, \omega)=f(x-h, s, \omega) R^{k}(x+h, s, \omega) \\
\left(A_{R}^{k}\right) f(x, h, s, \omega)=S^{k}(x-h, s, \omega) f(x+h, s, \omega)
\end{gathered}
$$

The adjoint derivative of $H(c) S$ with respect to $c$ must be computed, but this is generallly straightforward, as it involves only the formulae for a single step of depth extrapolation. The equations (5), (6) are to be solved in decreasing $k$ with $D S^{N_{z}}=D R^{N_{z}} \equiv 0$, in a loop over source index and frequency. The gradient at depth level $k$ is accumulated during this loop:

$$
\left(\nabla_{c} J\right)^{k}=\operatorname{Re}\left\{\sum_{s, \omega} D c^{k}\right\}
$$

\section{Inversion}

Successful optimization of the functions defined by equations (1) and (2) requires that the underlying mathematical structure of migration be respected. In particular, the ve- 
locities encountered during the iteration must remain smooth on the wavelength scale.

To enforce this smoothness, we use a B-spline representation based on a relatively coarse spacing of spline nodes. Let $m$ be a set of B-spline model parameters and $B$ the B-spline sampling operator (onto the image grid). Restriction to velocities of the form $c=B m$ gives a gradient in the spline parameters of the form

$$
\nabla_{m} J(B m)=B^{*} \nabla_{c} J(c)
$$

(here $J=J_{h}$ or $J_{\theta}$ ). We use a version of limited BFGS algorithm (Nocedal and Wright, 2000) to minimize $J$ as a function of $m$. Only $J$ and its gradient with respect $m$ is needed. We have now completely described the computation of these quantities.

\section{Synthetic examples}

We constructed data consistent with the model underlying DSVA by smoothing the Marmousi model using a lowpass filter that removes any length scale smaller than $25 \mathrm{~m}$. The difference between the original and smoothed models served as the reflectivity $\gamma$. Synthetic Born data is expressed via the downgoing one-way Green's functions of the smoothed velocity $G^{+}$via

$$
d(r, s, \omega)=\int G^{+}(x, s, \omega) G^{+}(x, r, \omega) \gamma(x) d x
$$

and can also be computed by solving a corresponding depth extrapolation problem. The simulation is made to acquire the same number of shots as the original Marmousi dataset: the source locations span uniformly from $2.625 \mathrm{~km}$ to $8.975 \mathrm{~km}$ at the spacing of $0.025 \mathrm{~km}$. The receiver arrays are fixed for each shot and cover the entire surface 
with spacing $0.01 \mathrm{~km}$. The migration is performed using frequencies from 3.3 to 40 $\mathrm{Hz}$ on square grids of $0.01 \mathrm{~km}$ each side. Note that use of this data in an inversion test commits an "inverse crime": the data completely agrees with the model on which the inversion is based.

Fig.12 shows the initial velocity model used in the inversion, obtained by smoothing the Marmousi model using a B-spline fitting with length scale of $2.25 \mathrm{~km}$ by $1 \mathrm{~km}$ (horizontal by vertical), much coarser than the resolution required for accurate imaging (see Fig.8) of the Marmousi data set (Versteeg, 1993).

Forty seven iterations of BFGS resulted in the models displayed in Figures (13) and (15). The fault block structure in the middle region has emerged in both cases; the difference appear to be subtle. The image from offset domain DSVA (Figure (9)) is a very accurate rendition of the structure of the actual Marmousi model, in all important respects. The angle domain image (Figure (16)) is of distinctly lower quality. Comparison of angle gathers (Figures (14), (17)) leads to the same conclusion: offset domain DSVA appears to have been more successful, given the expended amount of computational effort. Note that the angle gather displayed in Figure (14) is a postprocess result.

The reason for this difference in performance lies in the numerical condition of the Hessian operator. The operator $P_{\theta}$ is of order $1 / 2$ (in $2 \mathrm{D}$ !), meaning that it scales Fourier components by the square root of frequency. The operator $P_{h}$, on the other hand, is bounded, i.e. does not enhance high frequency components. As a result, the Hessian (second derivative) operator of $J_{h}$ is better conditioned than the Hessian of $J_{\theta}$ (finite vs. infinite condition). Convergence for Newton-like methods is heavily 
influenced by Hessian condition (Nocedal and Wright, 2000).

\section{Modified scheme}

The mathematical underpinning of DSVA is the same as that of prestack depth migration, namely the asymptotic theory of single or Born scattering about transparent (smooth) background models (Symes, 2008). Unsurprisingly, the behaviour of the algorithm degenerates as the wave propagation regime moves away from the theoretically justified arena for DSVA. A full waveform simulation of a reflection survey over the original Marmousi model (Figure(18)) provides a good example of this deterioration in DSVA performance. We used full accoustic waveform simulation (rather than demigration or forward Born simulation) to generate data with the same acquisition parameters as in the previous section. Precisely the same inititial model as shown in Figure(12) has been used to perform DSVA on this rough Marmousi data, but with substantially finer attempted resolution, in an effort to estimate velocity features not much larger than a wavelength. Velocity models with substantial variance at wavelength and near-wavelength scales are not transparent, and the theoretical justification for focussing-driven updates is in doubt. Indeed, the DSVA velocity estimates deviate significantly from the true model: they are contaminated by many strong artifacts (Figure(20),(21)), and do not produce particularly focused gathers. Note that multiple reflection does not appear to be the cause of this pathology: this model, over the time interval ( $3 \mathrm{~s}$ ) of simulation, does not produce substantial multiply reflected energy. Instead, the source of the misbehaviour is the attempt to extend the DSVA method to 
estimate near-wavelength scale heterogeneities, which are not transparent but which are necessary to fully explain the kinematics of single scattering for this model.

On the other hand, the longer-scale structure of these models is improved over that of the initial estimate, which suggests a possible modification of DSVA to extend its domain of validity, by combining the differential semblance objective with one based on image power. In contrast to DSVA, image power maximization makes constructive velocity updates only when the initial velocity is reasonably accurate. However velocities estimated by maximizing image power are relatively insensitive to noise: image power is strongly peaked at kinematically accurate velocities. Maximization of image power was explored by Toldi (Toldi, 1989), and more recently by Soubaras (Soubaras and Gratacos, 2007), who illustrates the robustness of the method by application to field data.

These observations suggest that DSVA might be combined with image power analysis to yield a method with the robust global convergence of DSVA (not trapped by local minima far from an optimal velocity) and the robust local convergence of image power analysis (not trapped by local mimina close - but not close enough - to optimal). Such a combined method has been implemented by Chauris (Chauris and Noble, 2001) using common offset Kirchhoff migration as the underlying imaging method. The first author has introduced a similar method in the context of depth extrapolation migration (Shen and Calandra, 2005).

Motivated by these considerations, we introduce the modified DSVA objective:

$$
J=\frac{1}{2}\|h I(x, h)\|^{2}-\frac{\beta^{2}}{2}\|I(x, 0)\| .
$$

Notice the imaging power term $\beta I(x, 0), \beta>0$ contains no information of image other 
than $h=0$, whereas the original DSVA term contains all information of image except at $h=0$. The combination produces a complete coverage of information of image in all offsets computed.

We compute gradient of the modified objective function using the same adjoint state approach explained above for pure DSVA. All of the formulas explained before hold with one small modification: we need only redefine the image residual to be

$$
D I=h^{2} I(x, h)-\beta^{2} I(x, 0)
$$

We applied this modified DSVA method to the rough Marmousi data with the same starting model (Figure(12)) and the same set of parameters associated with the differential semblance term. Stably converged results are shown for 49 and 99 iterations (Figures (22) (23), (24), 25)). The additional robustness of the image power term, which is active when the model is close to kinematically correct, leads to a rather precise final velocity estimate and very satisfactory image.

\section{Real data examples}

We have applied this implementation of modified DSVA to a segment of an 2D marine seismic line which covers a distance of about $97 \mathrm{kft}$. The preprocessing includes first arrival removal and tau-p deconvolution-based free surface multiple removal. Surface offsets range from $900 \mathrm{ft}$ to about $10,000 \mathrm{ft}$ with $81 \mathrm{ft}$ source and receiver spacing. The challenge of this data lies in the middle of the model where a vertical narrow gas cloud is expected. As shown in Figure (27), when migration with a $v(z)$ velocity model produces an artificial image sag. 
A ray-based reflection tomography velocity model was obtained (Figure (28) using this data set. The migrated image (Figure (30) is more interpretable than that obtained using the $v(z)$ velocity (Figure 27). One of the interpretation concerns remains, however: the shape of the low velocity zone is inconsistent with the surrounding geological setting.

Before we started the DSVA run stream, we have applied a source plane-wave phase encoding to this data. With 60 plane waves, the data is reduced to $20 \%$ of its original size (in frequency representation). The speed up by a factor of 5 is a significant advantage, in view of the repeated migrations required for BFGS iterations. The image of plane-wave migration shows virtually no visible difference compared to shot-record migration under the same one-way propagator. The offset common image gathers and DSVA gradient are produced in the same fashion as in shot-record migration except that the shot-record is replaced by plane-wave-records. We conducted 20 iterations of modified DSVA updates in offset domain with a (steepest descent) restart at the 6th iteration. The starting velocity as shown in Figure (26), is linearly increasing in depth. We chose this starting model in order to test the robustness of DSO scheme when starting model is reasonably far from the expected realistic model. In terms of compactness of the gas cloud, the velocity model obtained from the optimization noticeably agrees more with the geological interpretation (Figure (28) and Figure (29)). Moreover, the images below the gas cloud (Figure (31)) show more continuity compared with the tomography result (Figure (30)).

Once the image gathers in offset are computed, a slant stack (Sava and Fomel, 2003) can be applied to convert them to angle gathers. In general, the flatter the event 
the closer the velocity model approaches the true one. Figures (32)-(34) demonstrate increased degree of flatness in angle gathers from the image obtained at $v(z)$ velocity, tomography velocity to modified DSVA optimized velocity. An aparant gap in the vicinities of zero degree for each angle gather reflects the missing of zero (surface) offset in data.

\section{Conclusion}

Both offset and angle domain versions of DSVA are effective in updating a complex velocity model involving strong refraction, though the offset domain variant as presented here is somewhat more computationally efficient. Either supplementing the operator $P_{\theta}$ with a negative order factor, for $2 \mathrm{D}$, or computing in $3 \mathrm{D}$ where $P_{\theta}$ is bounded, would likely remove the comparative advantage of the offset domain computation.

The first test demonstrated here used "perfect" data, that is, data corresponding precisely to the theory underlying DSVA. It achieve nearly perfect results. The second test, which transgressed the assumed scale dichotomy underlying the theory, revealed a limitation of DSVA: even absent multiply reflected energy, the method on its own has limited ability to resolve rapidly changing velocity features of kinematic importance. We showed how to adjoin an image power term to the DSVA objective function; the modified DSVA method so obtained exhibits considerably more stability in the presence of important short-scale velocity variation.

Finally, we applied the modified DSVA method to a gas sag problem for which a reflection tomography velocity estimate is available. The modified DSVA result is 
more kinematically consistent with the data (more focused image gathers) than is the reflection tomography result, and yields a more interpretable image.

Many open questions remain concerning the sensitivity of the approach to data imperfections, on the one hand, and the possibility of similar approaches based on more sophisticated modeling, on the other. Previous work has shown that DSVA is quite sensitive to the presence of multiply reflected energy (eg. (Verm and Symes, 2006)). This is hardly surprising, as the method is relies for its theoretical justification on the single scattering assumption. Various methods have been proposed to reduce the sensitivity of DSVA to multiples and other coherent noise (Gockenbach and Symes, 1999; Mulder and ten Kroode, 2002a; Li and Symes, 2007). The ultimate remedy may lie in inclusion of multiple reflection in the underlying model of wave propagation see Symes (Symes, 2008) for a suggestion along these lines.

\section{Acknowledgement}

This work was partly supported by The Rice Inversion Project. The author wish to thank Scott Morton for his extensive advice and many valuble suggestions, and Biondo

Biondi, Maarten de Hoop, Christiaan Stolk, and Richard Verm for many useful and inspiring discussions on the topics of seismic inversion and velocity analysis.

\section{References}

Chauris, H. and M. Noble, 2001, Two-dimensional velocity macro model estimation from seismic reflection data by local differential semblance optimization: applica- 
tions synthetic and real data sets: Geophys. J. Int., 144, 14-26.

Foss, S. k., B. Ursin, and M. V. de Hoop, 2004, Depth-consistent p - and s - wave velocity reflection tomography using pp and ps seismic data: Expanded Abstracts, Society of Exploration Geophysicists, 53rd Annual International Meeting, 23632367.

Gockenbach, M. and W. Symes, 1999, Coherent noise suppression in velocity inversion: 69th Annual International Meeting, Expanded Abstracts, 1719-1723, Society of Exploration Geophysicists.

Kabir, N., U. Albertin, M. Zhou, V. Nagassar, E. Kjos, and P. Whittaker, 2007, Use of refraction, reflection and wave-equation-based tomography for imaging beneath the shallow gas: a Trinidad field data example: 77th Annual International Meeting, Expanded Abstracts, 2812-2815, Society of Exploration Geophysicists.

Kern, M. and W. Symes, 1994, Inversion of reflection seismograms by differential semblance analysis: Algorithm structure and synthetic examples: Geophysical Prospecting, 565-614.

Li, J. and W. Symes, 2007, Interval velocity estimation via nmo-based differential semblance: Geophysics, 72, U75-U88.

Mulder, W. and A. ten Kroode, 2002a, Automatic velocity analysis by differential semblance optimization: Geophysics, 67, 1184-1191.

Mulder, W. A. and A. P. E. ten Kroode, 2002b, Automatic velocity analysis by differential semblance optimization: Geophysics, 67, 1184-1191.

Nocedal, J. and S. Wright, 2000, Numerical optimization: Springer Verlag. 
Sava, P. and S. Fomel, 2003, Angle domain common-image gathers by wavefield continuation methods: Geophysics, 68, 1065-1074.

Shen, P. and H. Calandra, 2005, One-way waveform inversion within the framwork of adjoint state differential migration: Expanded Abstracts, Society of Exploration Geophysicists, 75th Annual International Meeting (submitted).

Shen, P., C. Stolk, and W. Symes, 2003, Automatic velocity analysis by differential semblance optimization: Expanded Abstracts, Society of Exploration Geophysicists, 73rd Annual International Meeting, 2132-2135.

Soubaras, R. and B. Gratacos, 2006, Velocity model building by semblance maximization of modulated-shot gathers: 76th Annual International Meeting, Expanded Abstracts, SVIP1.3, Society of Exploration Geophysicists.

$\ldots$ _ 2007, Velocity model building by semblancemaximization of modulated-shot gathers: Geophysics, 72, U67.

Stolk, C. and W. Symes, 2004, Kinematic aritifacts in prestack depth migration: Geophysics, 69, 562-575.

Stolk, C. C., M. V. de hoop, and W. W. Symes, 2005, Kinematics of shot-geophone migration: Technical Report 05-04, Department of Computational and Applied Mathematics, Rice University, Houston, Texax, USA.

Symes, W., 1986, Stability and instability results for inverse problems in severaldimensional wave propagation in glowinski, r., and lions, j.: Proc. 7th International Conference on Computing Methods in Applied Science and Engineering:: NorthHolland, 69. 
Symes, W. and R. Versteeg, 1993, Velocity model determination using differential semblance optimization: expanded Abstracts, Society of Exploration Geophysicists, 63rd Annual International Meeting, 696-699.

Symes, W. W., 2008, Migration velocity analysis and waveform inversion: Geophysical Prospecting, 56, (in press).

Toldi, J., 1989, Velocity analysis without picking: Geophysics, 54, 191-199.

Verm, R. and W. Symes, 2006, Practice and pitfalls in NMO-based differential semblance velocity analysis: 75th Annual International Meeting, Expanded Abstracts, SI3.7, Society of Exploration Geophysicists.

Versteeg, R. J., 1993, Sensitivity of prestack depth migration to the velocity model: Geophysics, 58, 873-882. 


\section{Captions}

Figure(1). A smoothed version of the Marmousi velocity model, considered as a "true" model for this study. The horizontal velocity variability is kept at a length scale greater than 120 metesr.

Figure(2). A reflectivity distribution map $\gamma(x)$ obtained by high pass filtering the original Marmousi velocity model.

Figure(3). Data in time simulated by finite difference time stepping. Velocity used is the superposition of the background velocity and the reflectivity distribution, as shown in figure(1) and figure(2), respectively. Events beyond the first arrivals are muted.

Figure(4). Data in time simulated by the one-way propagator according to equation(7) with reflecting boundary condition. Events beyond the direct arrivals are muted. Although the amplitudes do not agree with that obtained from the time simulation, the reflection events are modeled at the correct time.

Figure(5). The image obtained through a perfect pre-stack depth migration at the "true" velocity as shown in figure(1), where the migration propagator completely agrees with the modeling propagator.

Figure(6). The migrated offset gathers, picked from the center of the model, obtained through pre-stack depth migration at the "true" velocity as shown in figure(1). The 
offset ranges from $-0.5 \mathrm{~km}$ to $0.5 \mathrm{~km}$ with an interval of $0.01 \mathrm{~km}$ for each gather. The energy is clearly seen to be concentrated, to the leading order, at the center of the gather.

Figure(7). The angle gathers obtained through a "Fomel/Sava" Radon transform from the offset gathers as shown in figure(6). The flatness angle indicates an alternative measurement of the accuracy of the velocity as oppose to the concentration in offset.

Figure(12). The starting velocity model obtained by the B-spline projection corresponds to a horizontal length scale of $1.3 \mathrm{~km}$.

Figure(8). The initial image at the starting velocity model. Significant distortion of the image, particularly in the deeper part of the model, is due the presence of large velocity error.

Figure(10). The initial offset gather at the starting velocity model. Although the data is strictly one-way Born, the energy is disperssive across the range of offsets modeled, indicating the missing of strong refracting structures in the starting model.

Figure(9). Image by optimized velocity of offset domain DSVA at 47th iteration.

Figure(13). Optimized velocity by offset domain DSVA at 47th iteration.

Figure(11). Offset gathers by offset domain DSVA at 47 th iteration. They are well 
focused at $h=0$.

Figure(14). The angle gathers obtained through a "Fomel/Sava" Radon transform from the optimized offset gathers as shown in figure(11). As the offset gathers become focused, the corresponding angle gathers become flat.

Figure(16). The image obtained by the angle domain DSVA. The image shows more distortion compared with that of the offset domain DSVA (figure(9)).

Figure(15). The optimized velocity through angle domain DSVA. It shows more variabilities compared with figure(13).

Figure(17). The angle gathers through angle domain DSVA. It is clear that they are not as flat in comparison with those obtained from the offset domain DSVA (figure(14)).

Figure(19). Initial image at the $v(z)$ velocity model (figure(12)). Although obtained at different data, similar features are shown compared to figure(8).

Figure(20). Velocity output of DSVA to rough Marmousi data at 49th interation. The inherited roughness of velocity in the data makes the optmization problem more difficult.

Figure(21). Velocity output of DSVA to rough Marmousi data at 82th interation. Fea- 
tures of the correct Marmousi velocity model are burried under many artifacts. As the number of iterations increase, these artifacts become stronger.

Figure(22). Velocity output of modifed DSVA at 49th interation. It shows close resemblance to the true Marmousi model. The imaging power term plays an important role to stablize the results.

Figure(23). Image output of modified DSVA at 49th interation. Compared to the initial image (figure(19)), significant coherent engergies are recollected for deeper images.

Figure(24). Velocity output of modifed DSVA at 99th interation. As the number of iterations increases, the velocity estimate kept stable.

Figure(25). Image output of modified DSVA at 99th interation. The image continues to improve as the optmization continues.

Figure(26). Initial $v(z)$ velocity model. To test the roubustness of DSVA, we take the average velocity of the first and the last layer from best the tomography velocity (figure(28)) and linearly interpolate for all depths to creat this $v(z)$ veloity model.

Figure(27). Image at the initial $v(z)$ velocity.

Figure(28). The optimized velocity output by ray tracing tomography which is per- 
formed independently. Vertical and horizontal units are 1000 feets.

Figure(29). The velocity output by modified offset domain DSVA at 20th iteration. The starting model is shown in figure(26). Vertical and horizontal units are 1000 feets.

Figure(30). Image obtained at the optimized velocity of ray tracing tomography.

Figure(31). Image by optimized velocity of modified offset domain DSVA.

Figure(32). Angle gathers by Radon transform from offset gathers at the initial $v(z)$ velocity model.

Figure(33). Angle gathers by Radon transform from offset gathers at the optimized velocity of ray tracing tomography.

Figure(34). Angle gathers by Radon transform from offset gathers at the velocity output of modified offset domain DSVA at 20th iteration. 


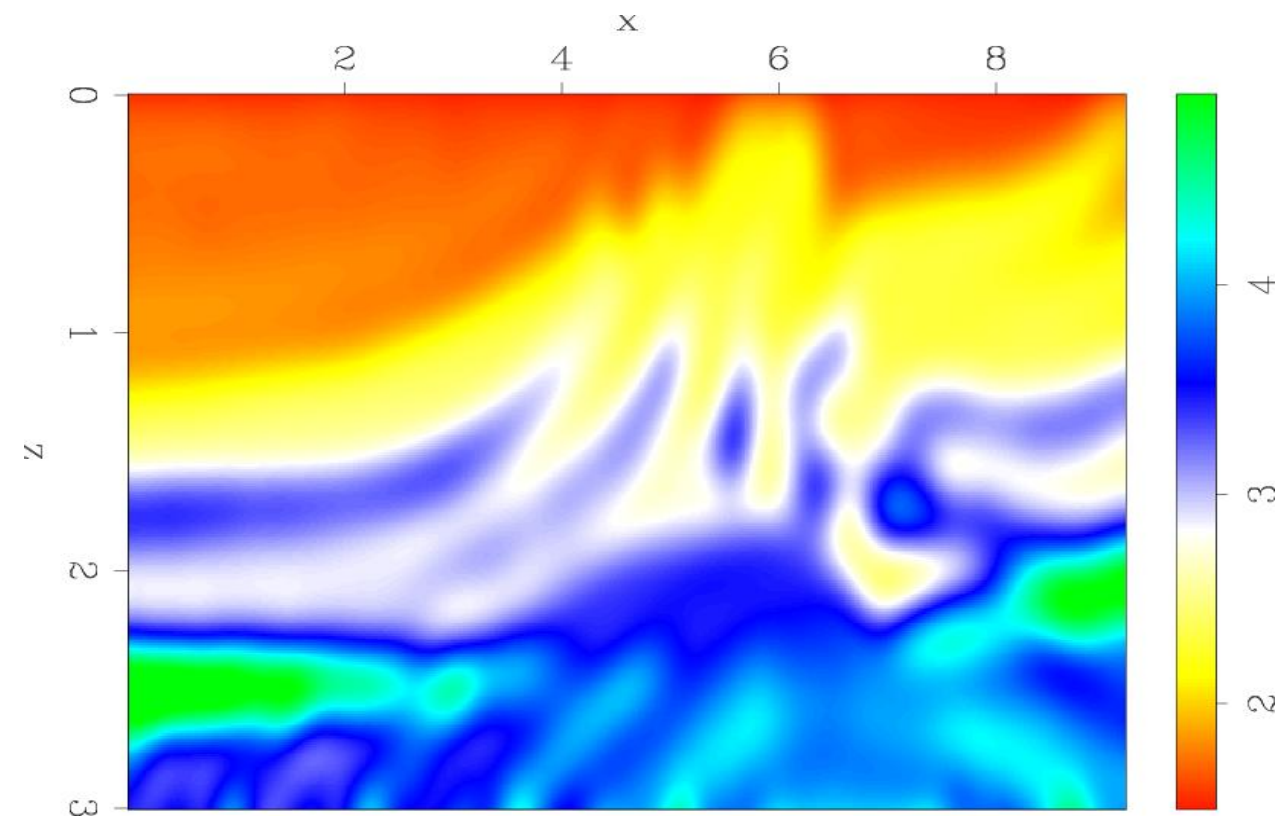

Fig. 1: Smoothed "true" velocity model.

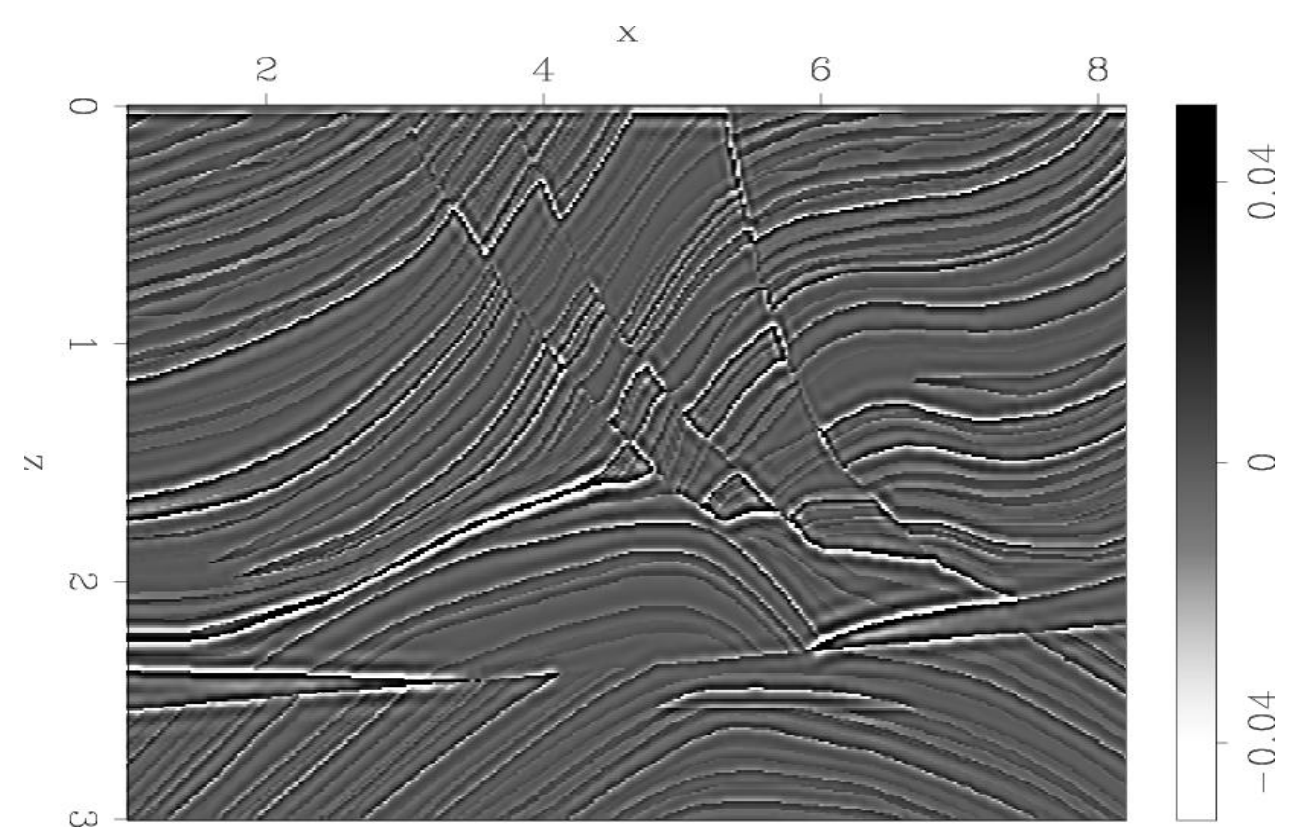

Fig. 2: Singular reflectivity model. 


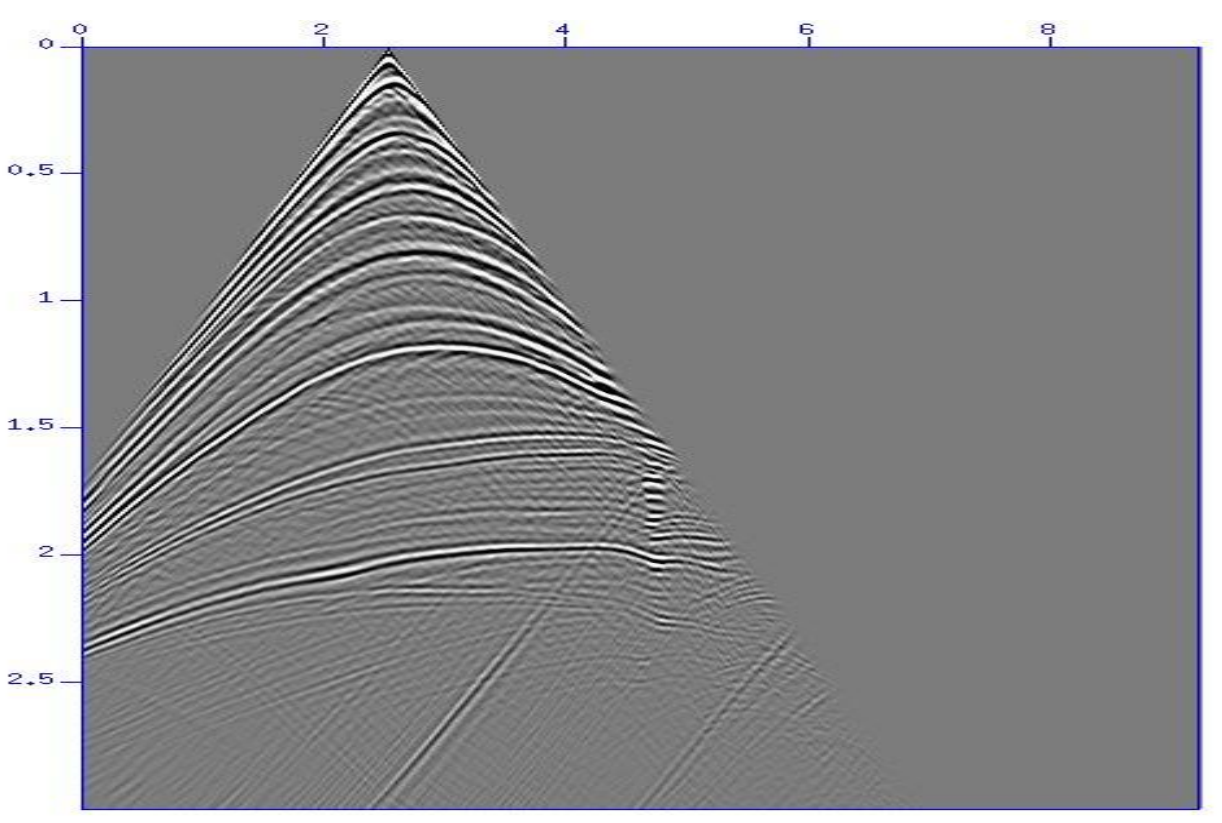

Fig. 3: Data by twoway simulation, direct arrival removed.

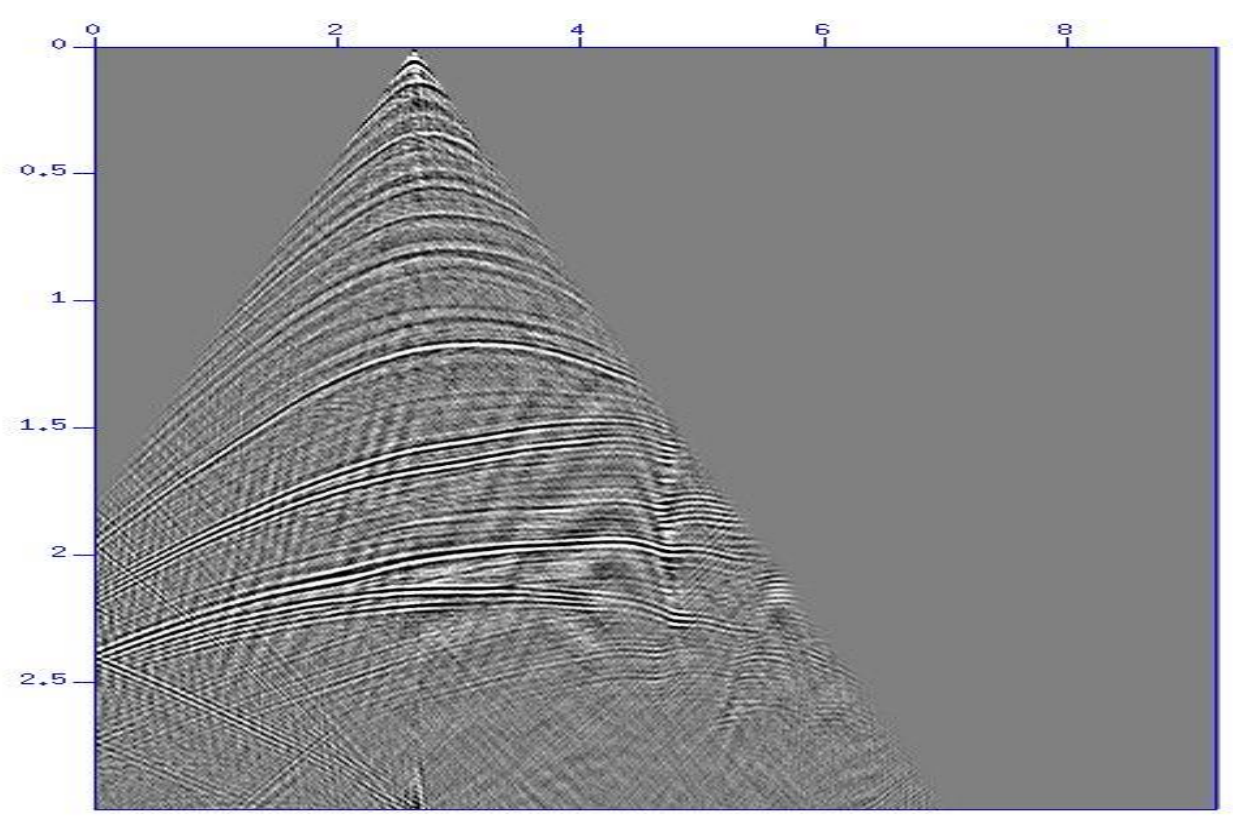

Fig. 4: Data by one-way simulation. 


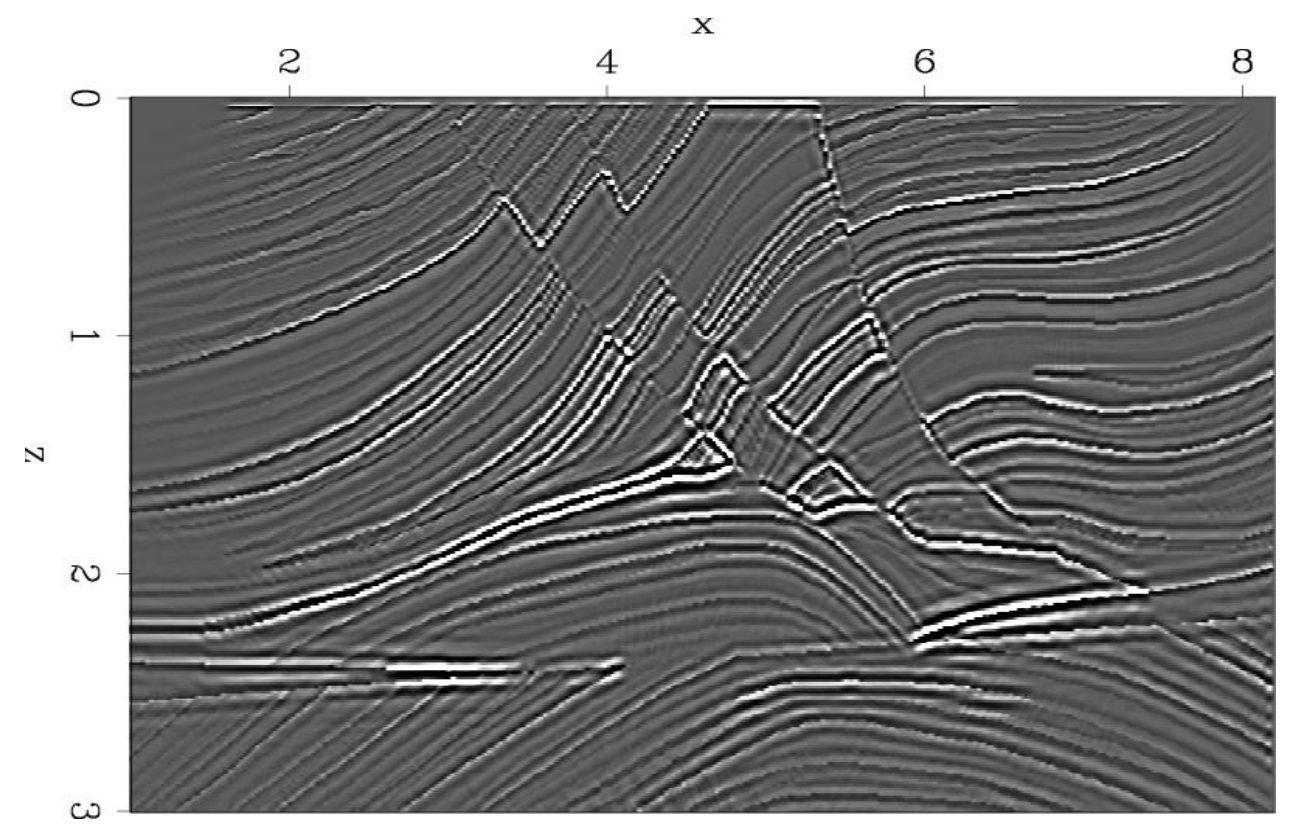

Fig. 5: Image at true velocity model.

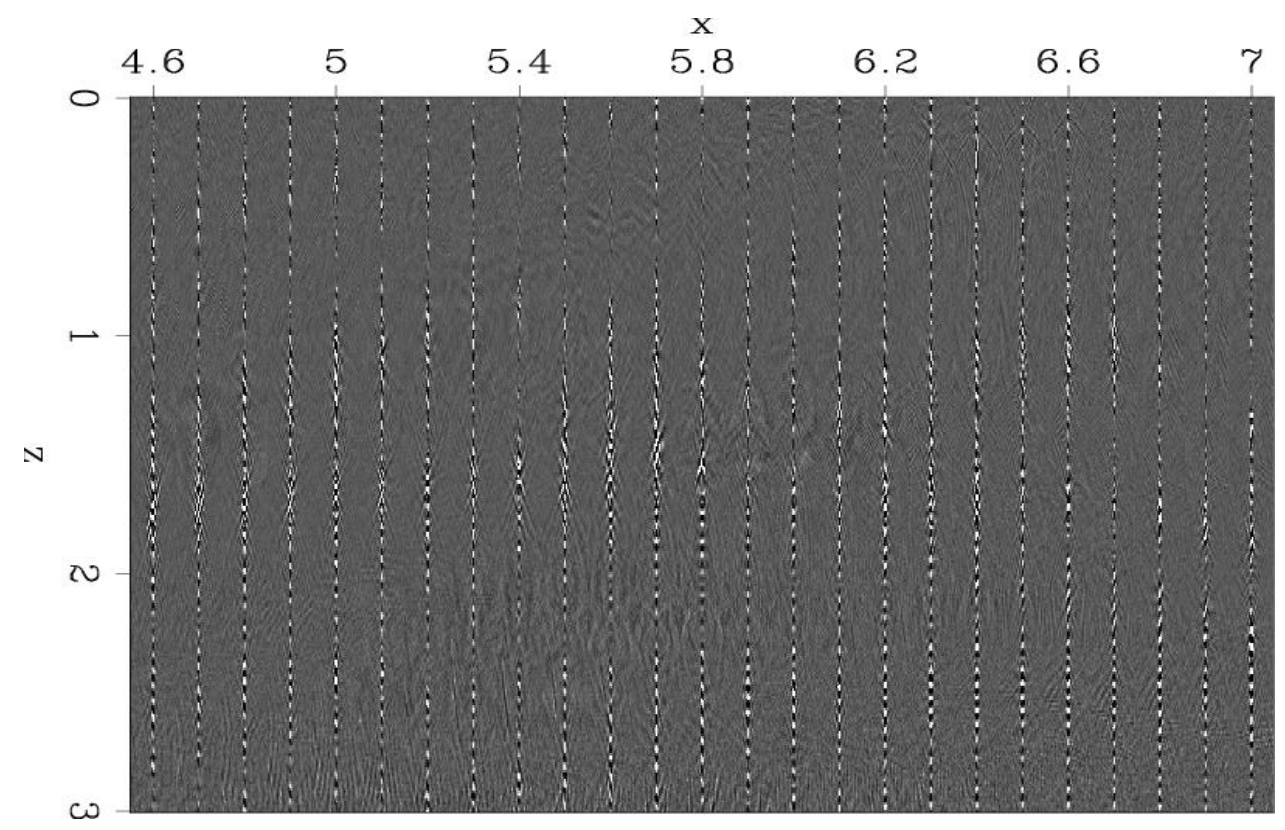

Fig. 6: Offset gathers obtained at the true velocity model. 


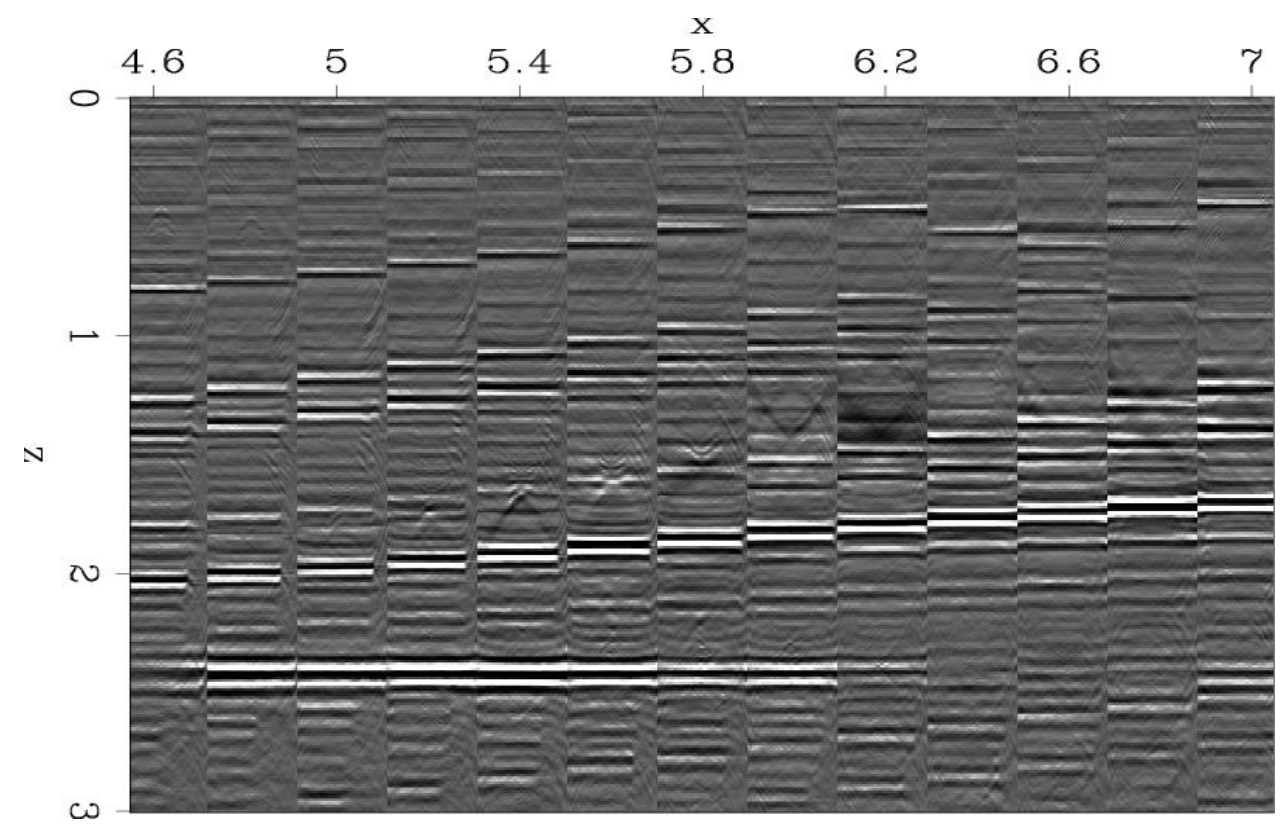

Fig. 7: Angle gathers at the true velocity model. 


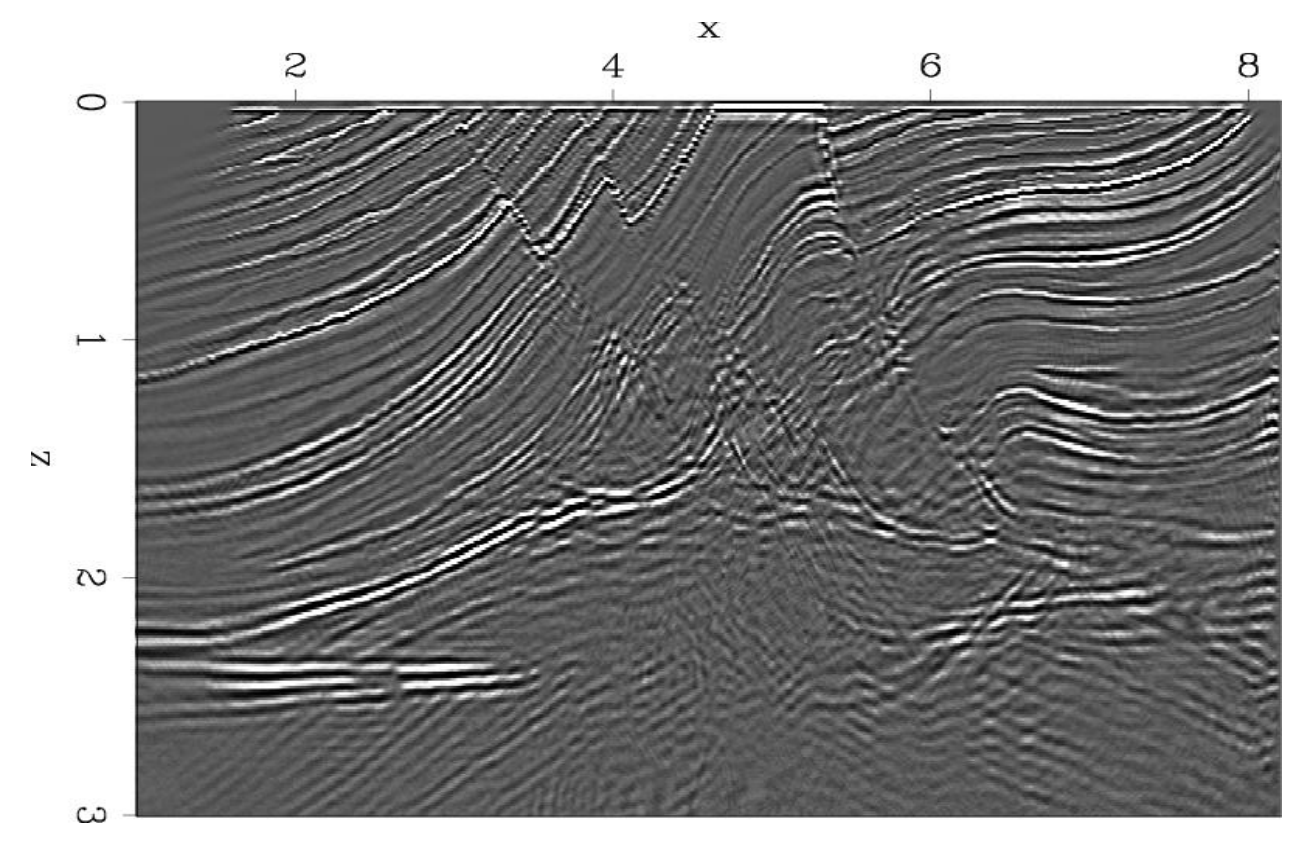

Fig. 8: Image obtained by the initial velocity model.

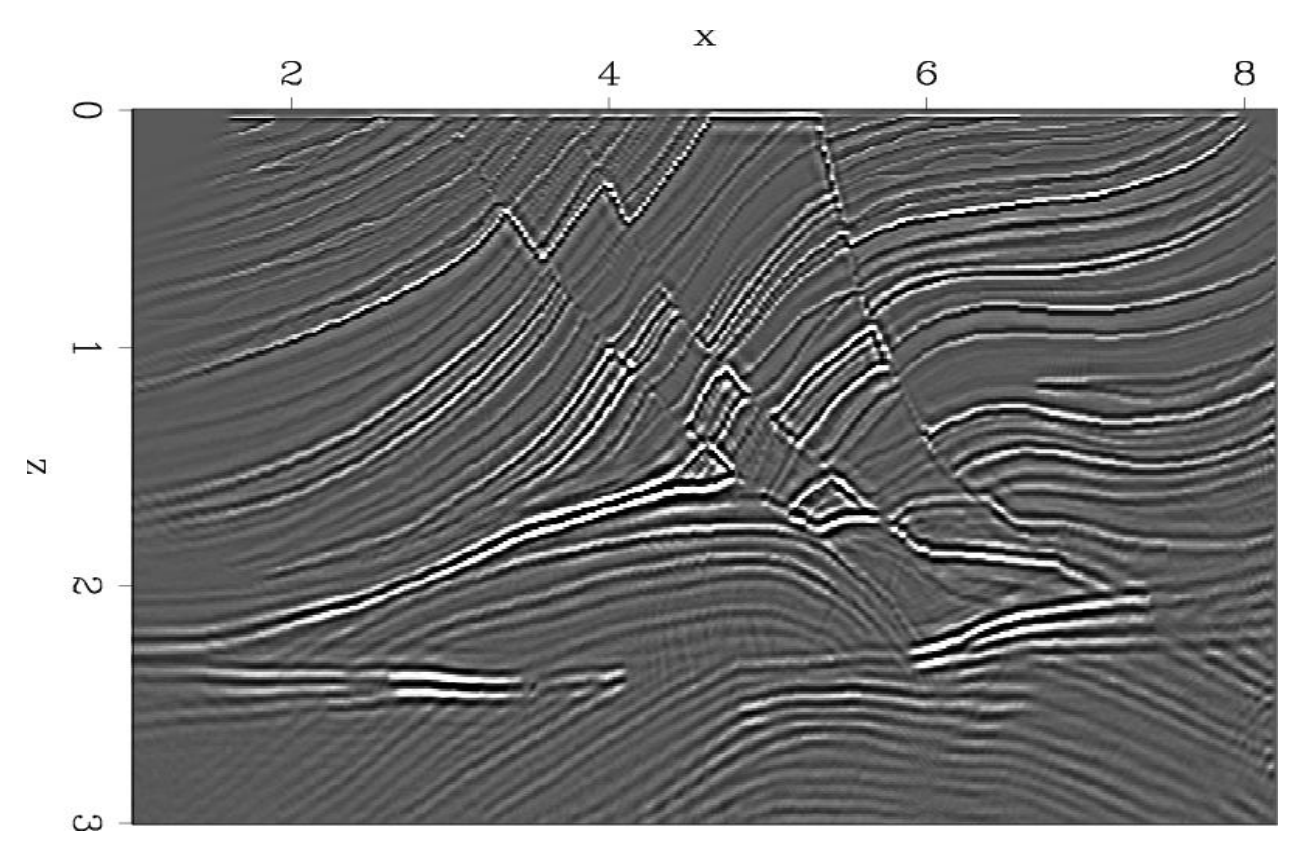

Fig. 9: Image obtained by the output velocity at 47 th iteration. 


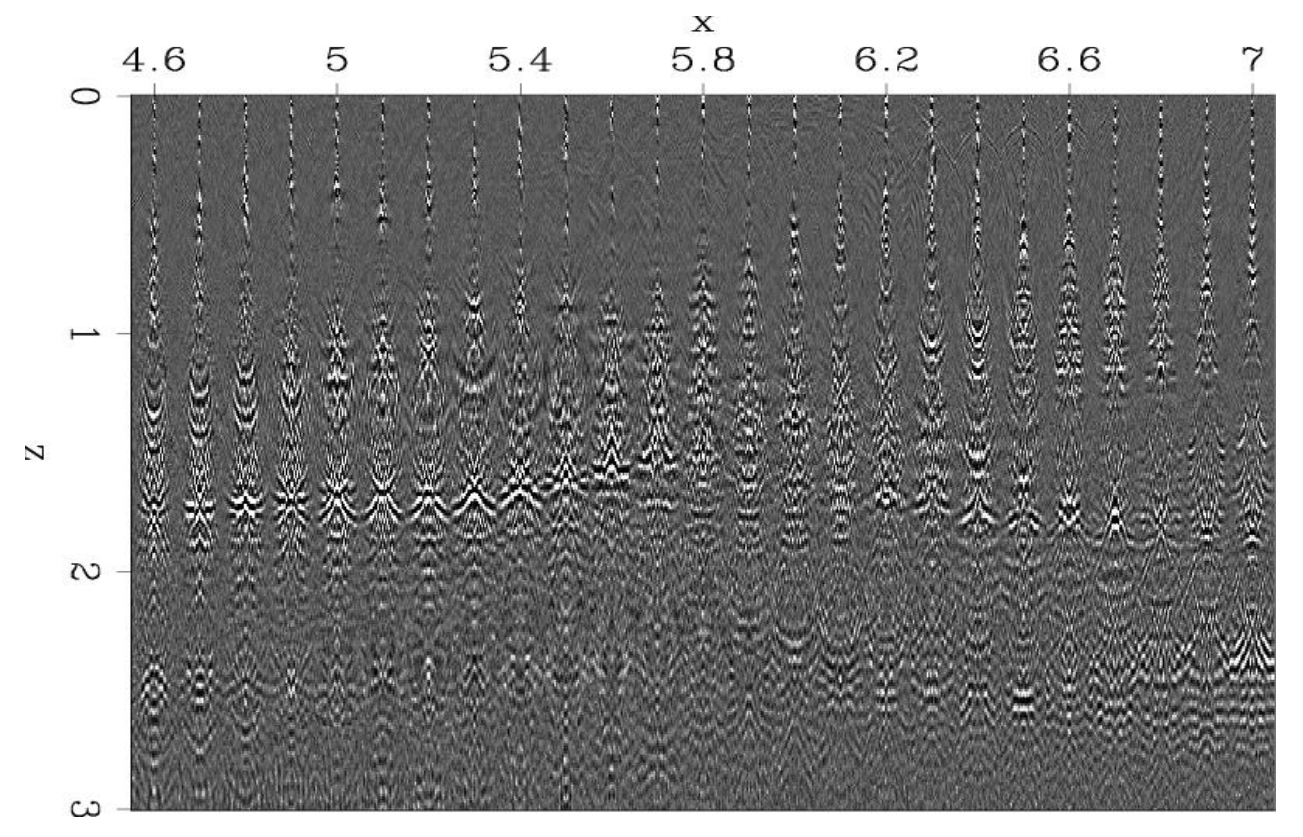

Fig. 10: Offset gathers obtained at the initial velocity model.

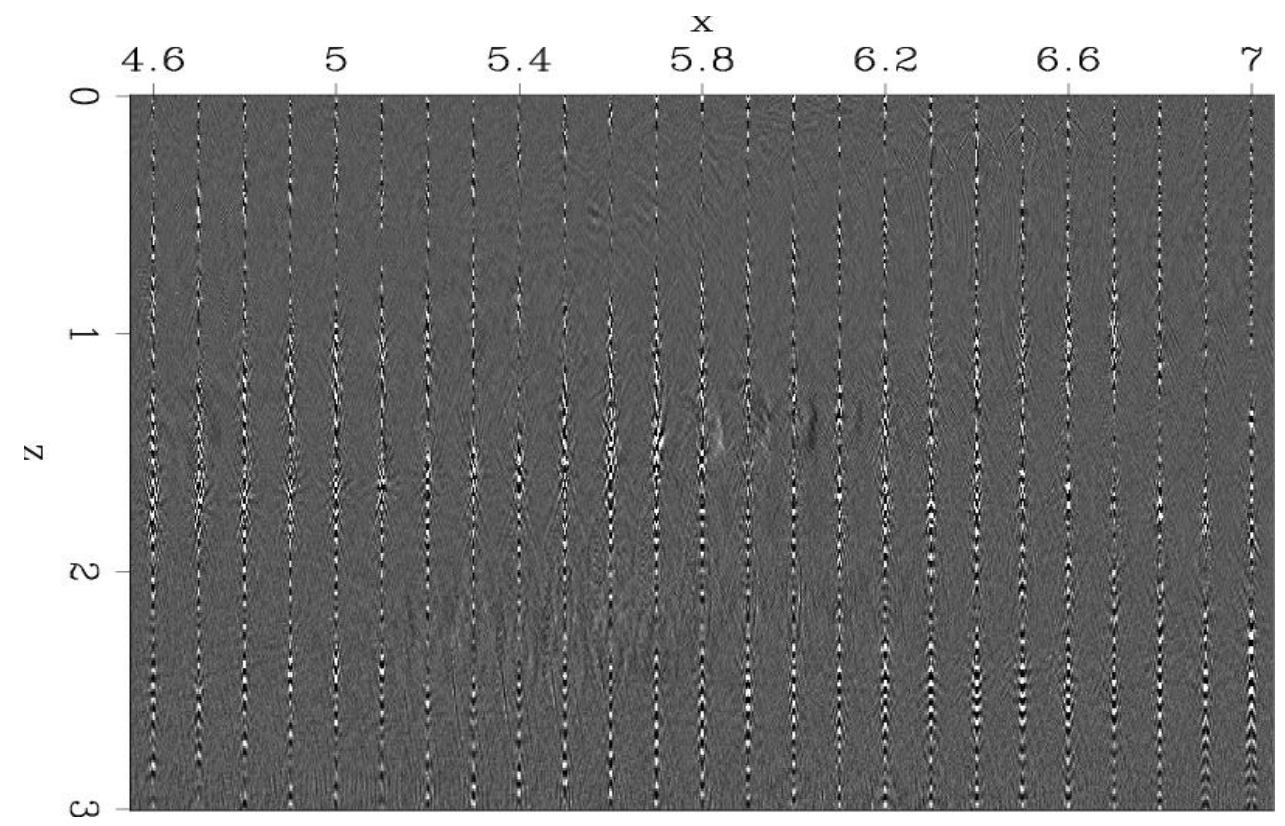

Fig. 11: Output of offset gathers at 47th iteration by offset domain DSVA. 


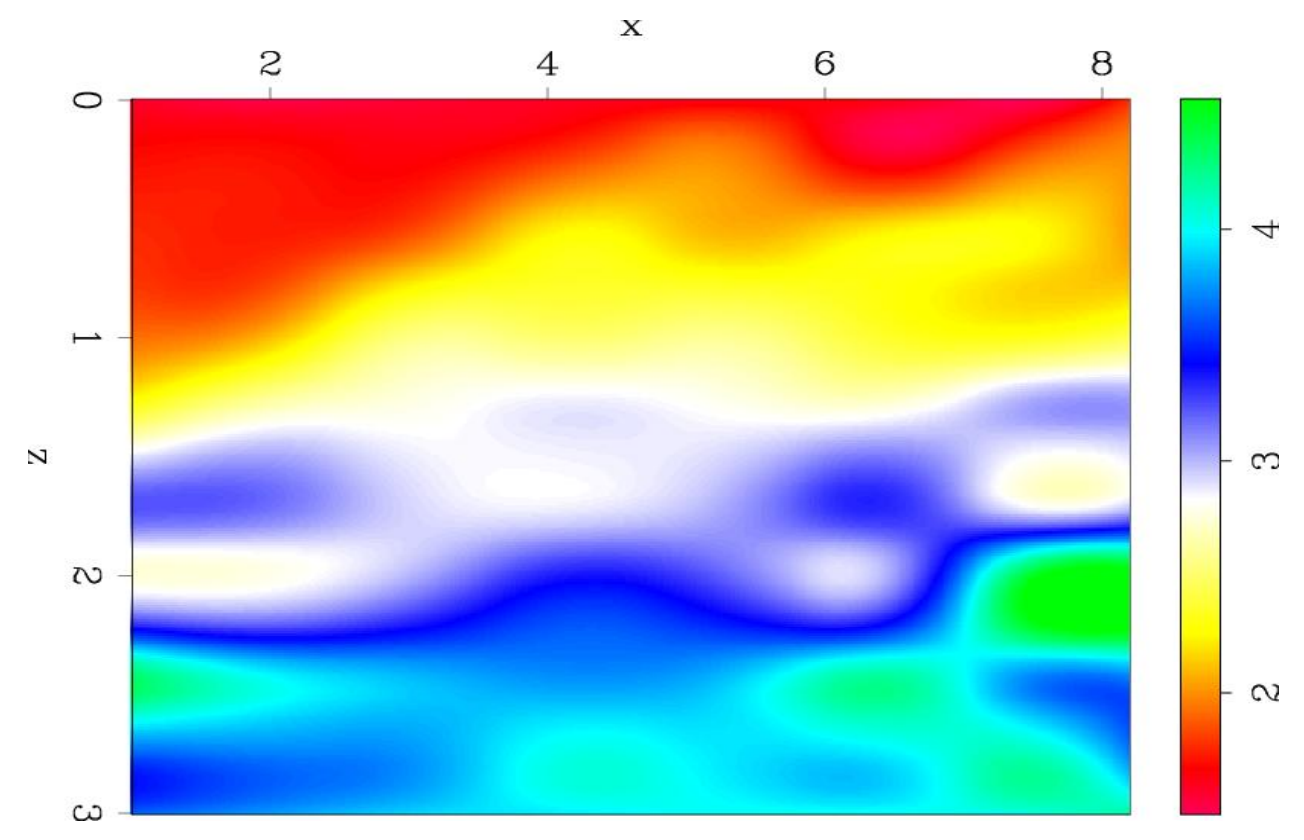

Fig. 12: Initial velocity model used for optmization.

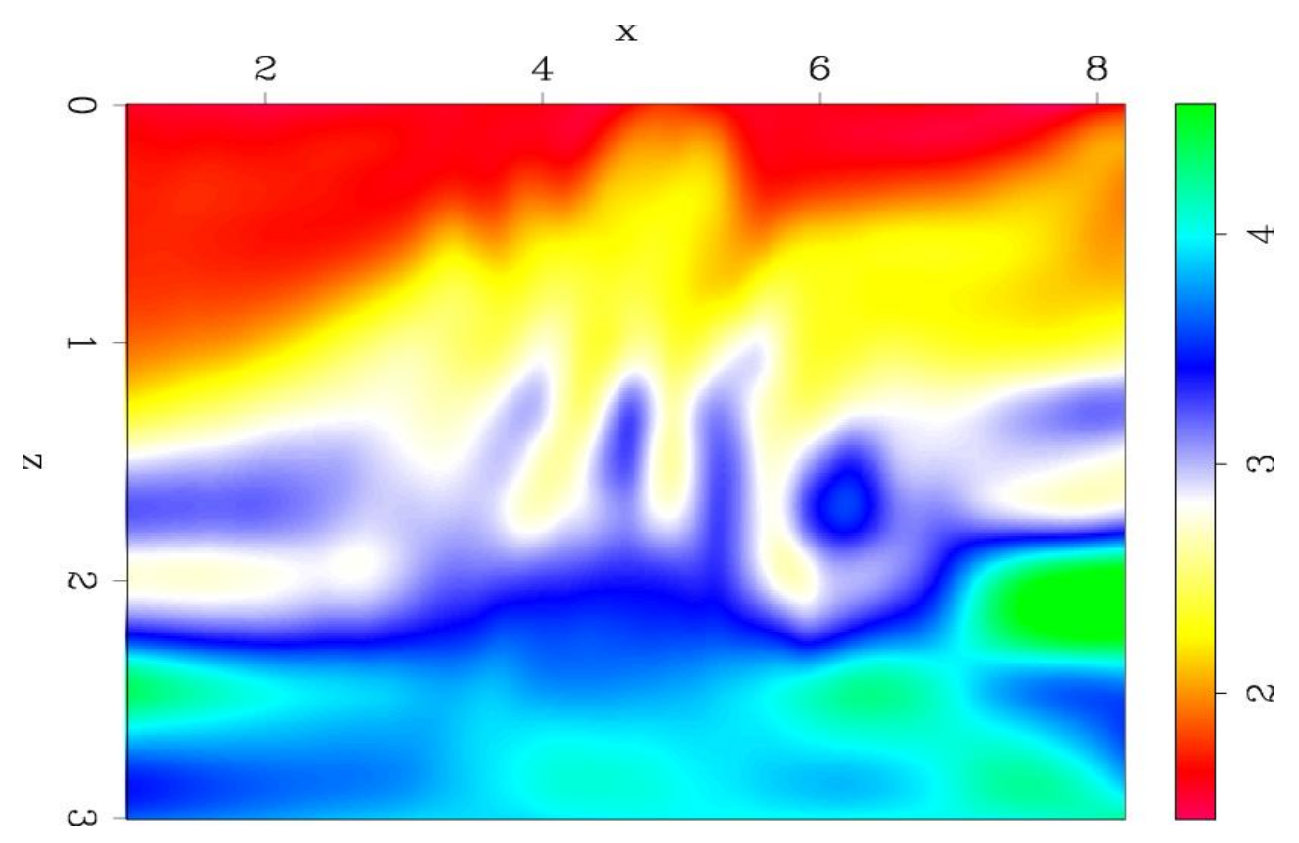

Fig. 13: Output velocity model at 47th iteration of offset domain DSVA. 


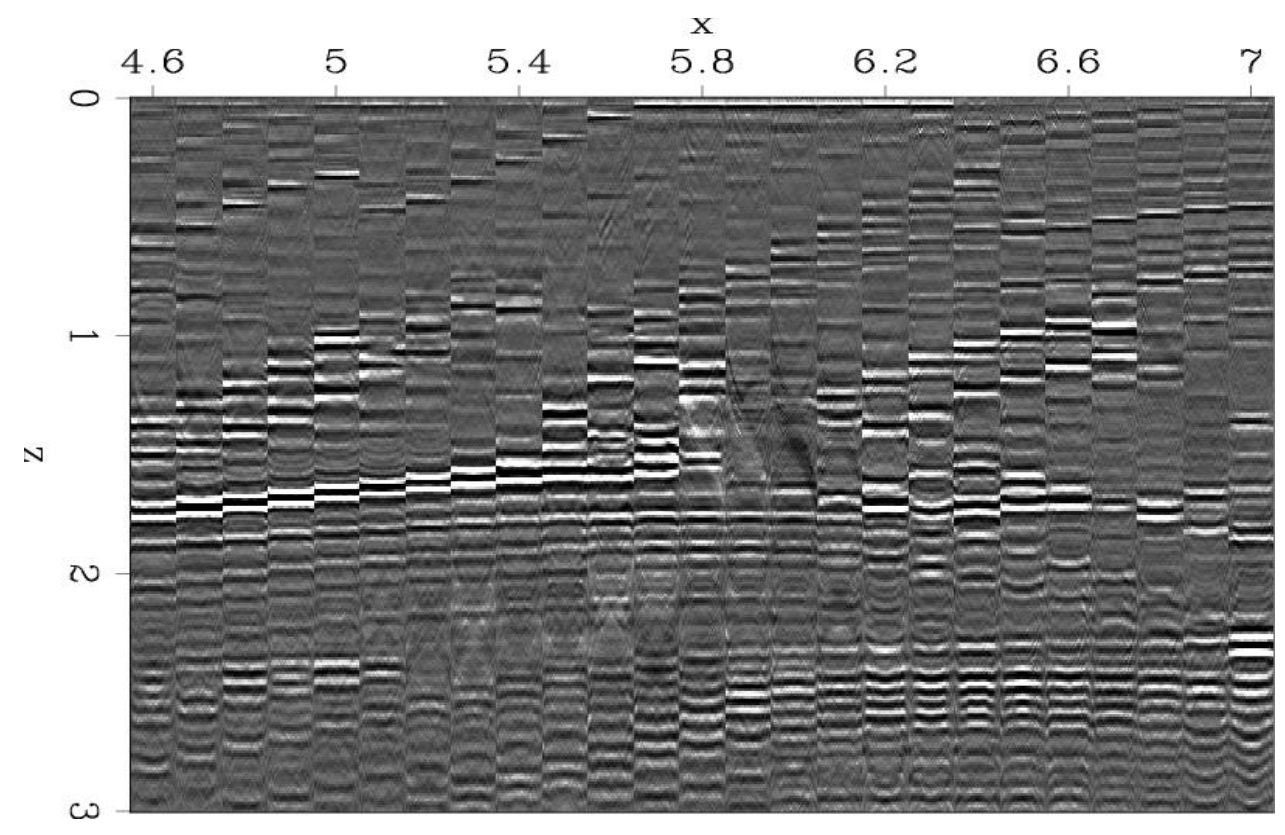

Fig. 14: Angle gathers by optmized velocity of offset domain DSVA. 


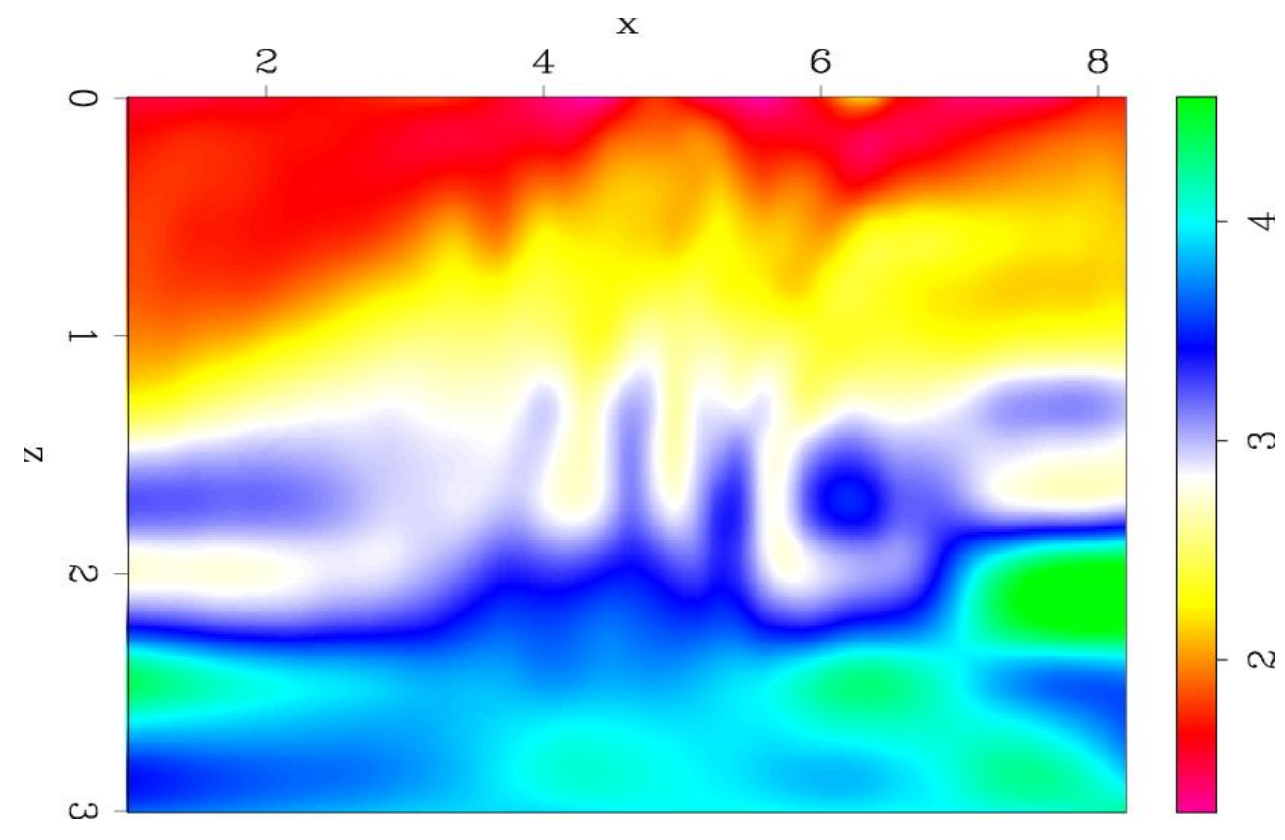

Fig. 15: Optimized velocity model by angle domain DSVA.

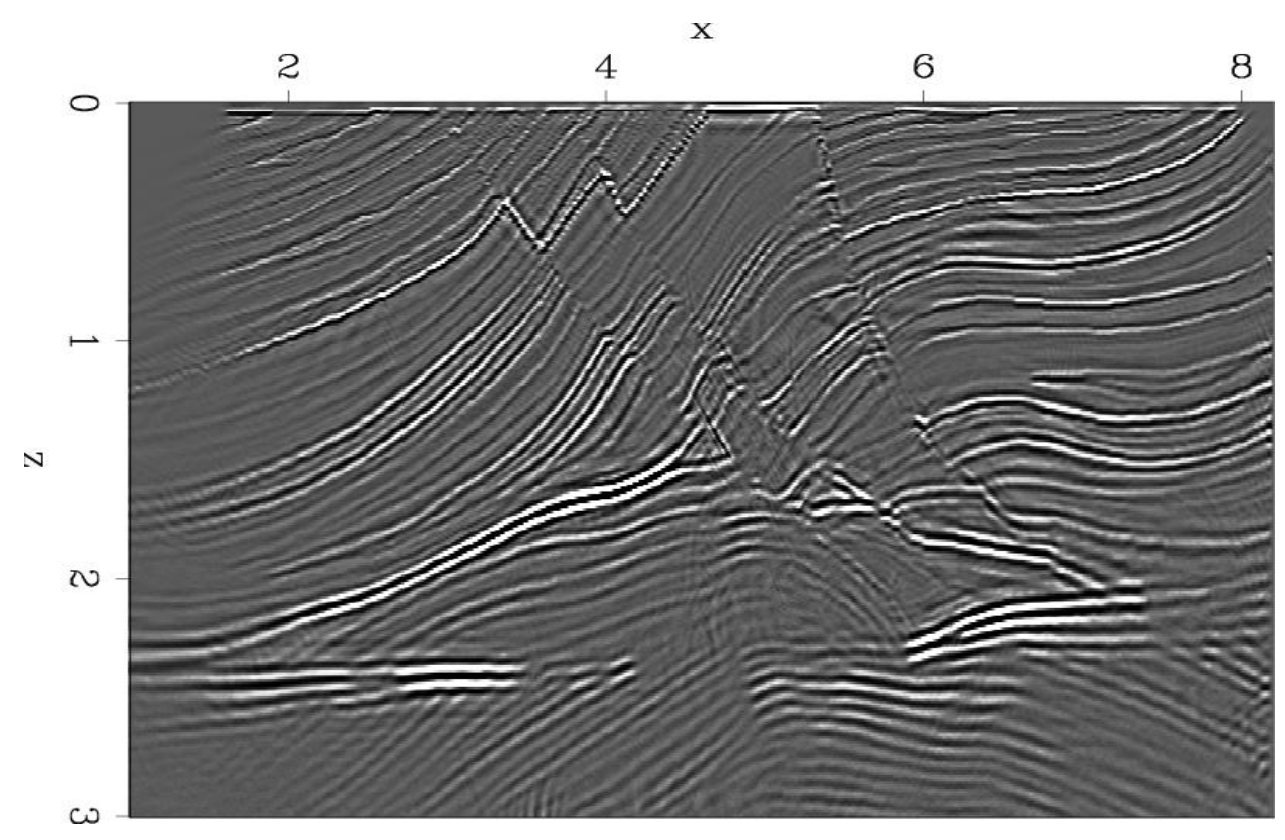

Fig. 16: Image by optmized velocity of angle domain DSVA. 


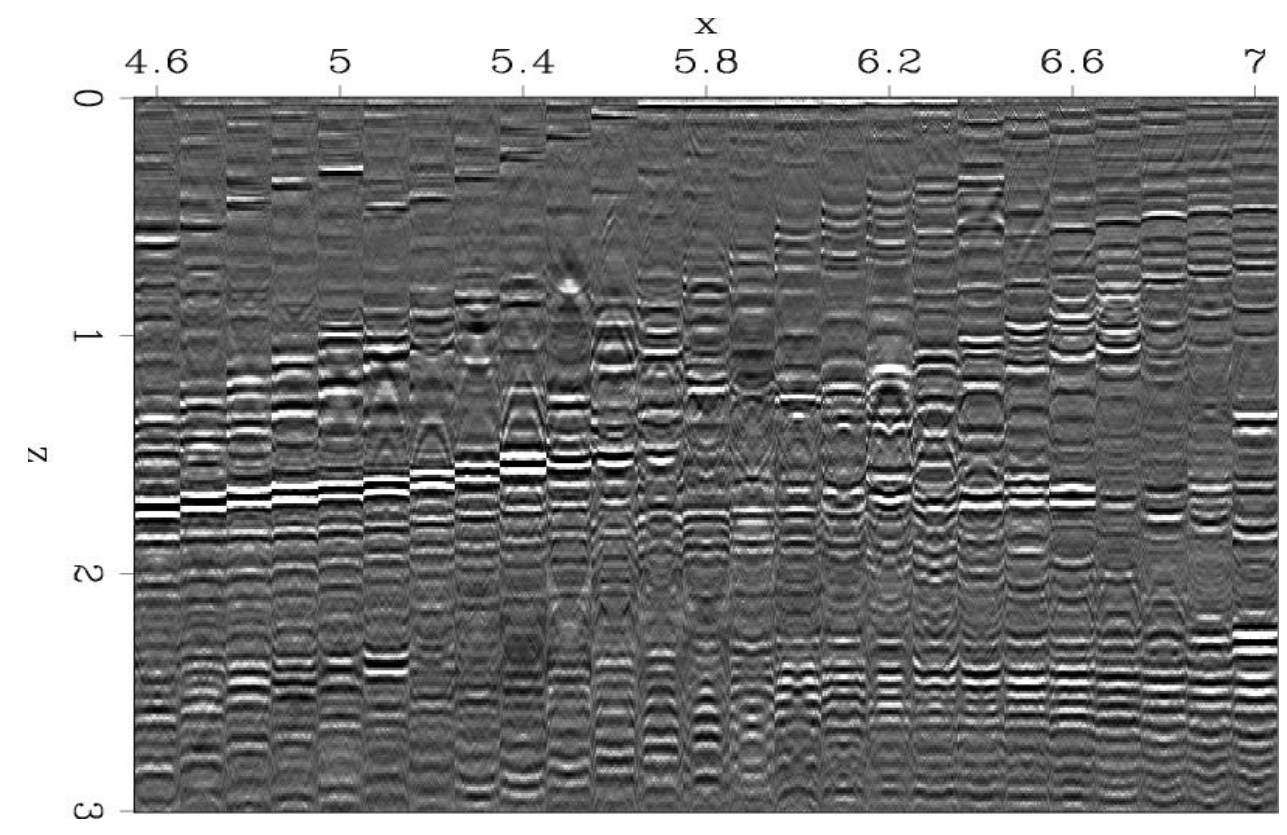

Fig. 17: Angle gathers by optimized velocity of angle domain DSVA. 


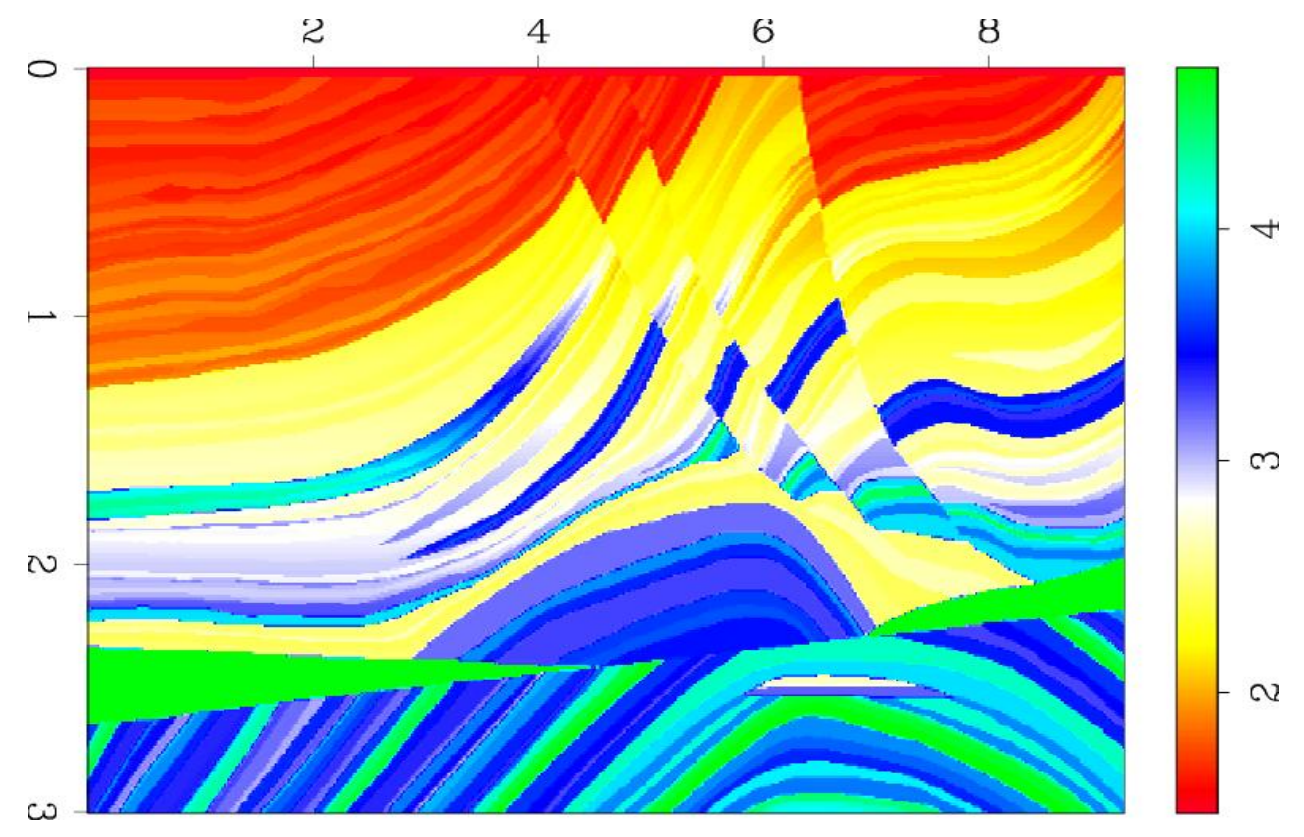

Fig. 18: Unsmoothed Marmousi velocity model.

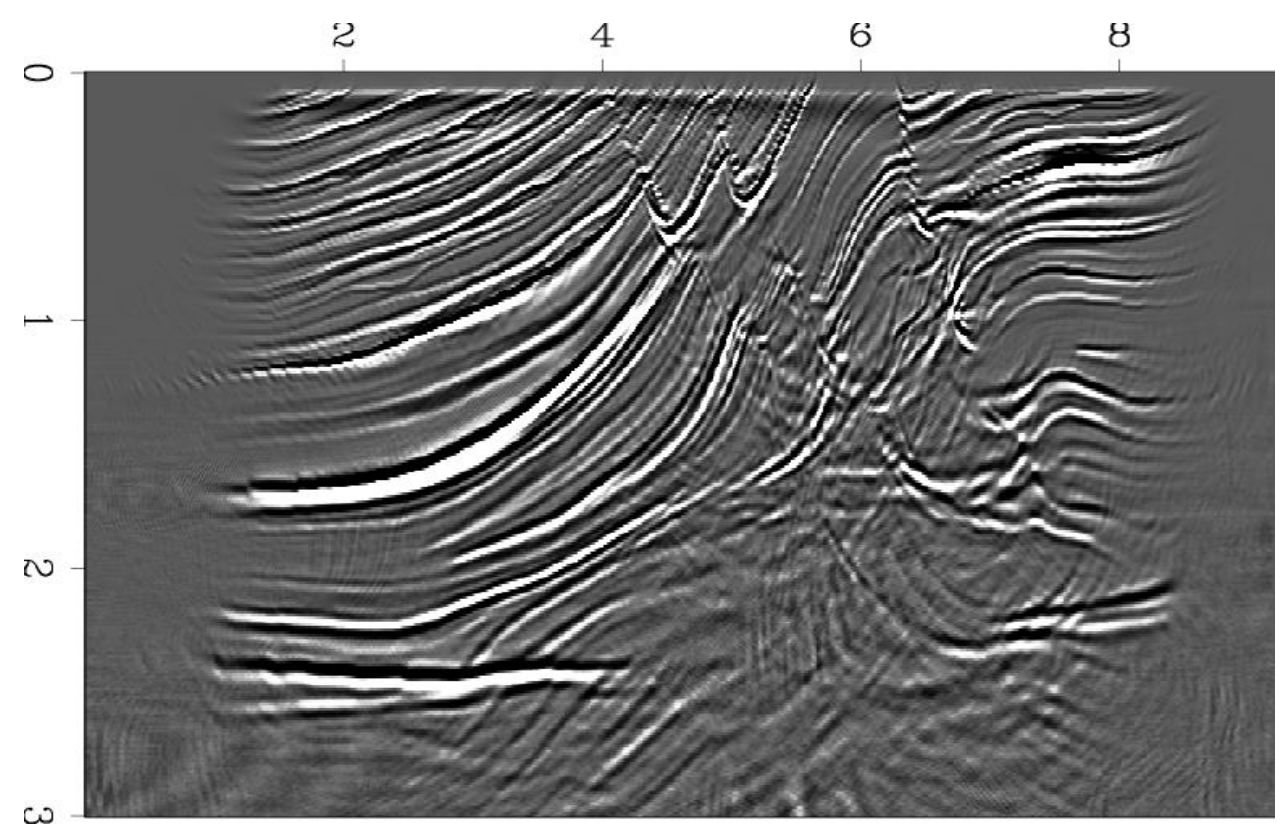

Fig. 19: Initial image velocity as shown in figure(12). 


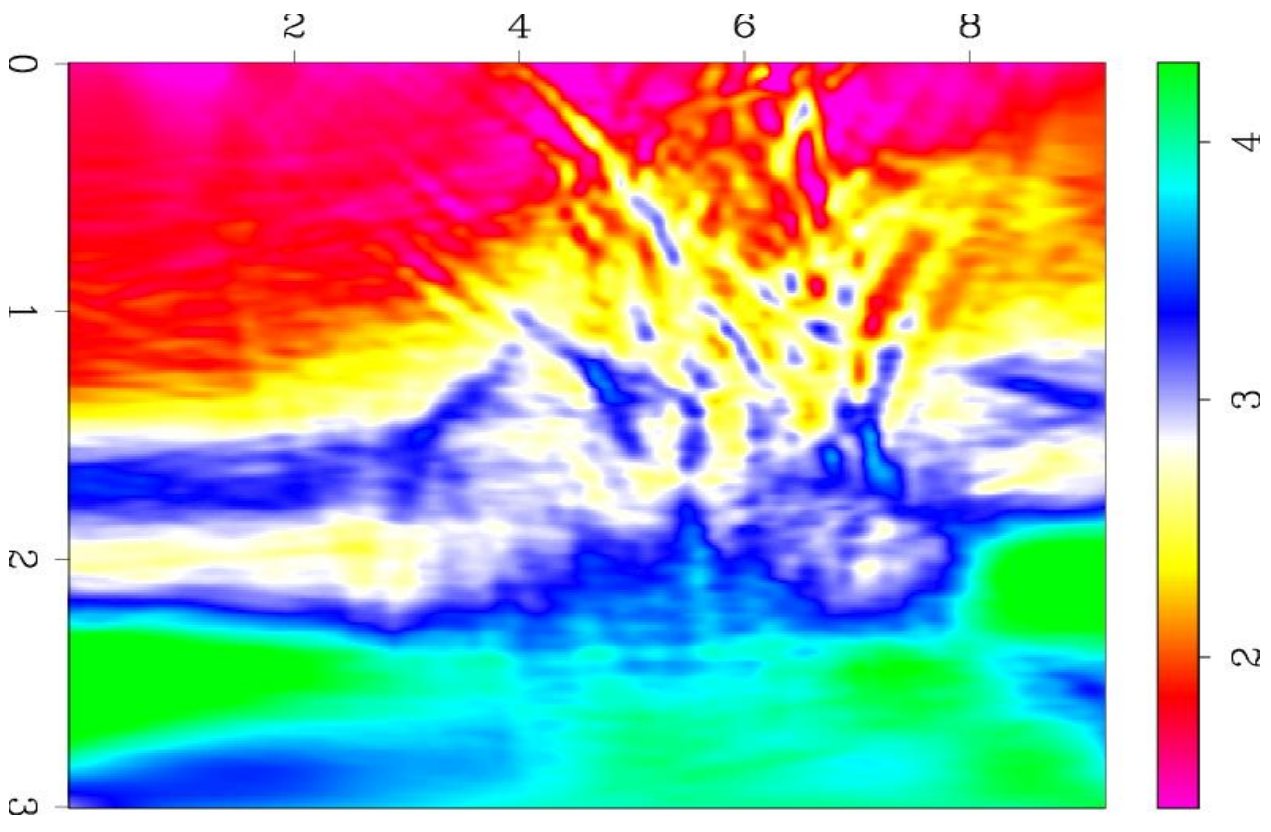

Fig. 20: Velocity output of DSVA to rough Marmousi data at 49th iteration

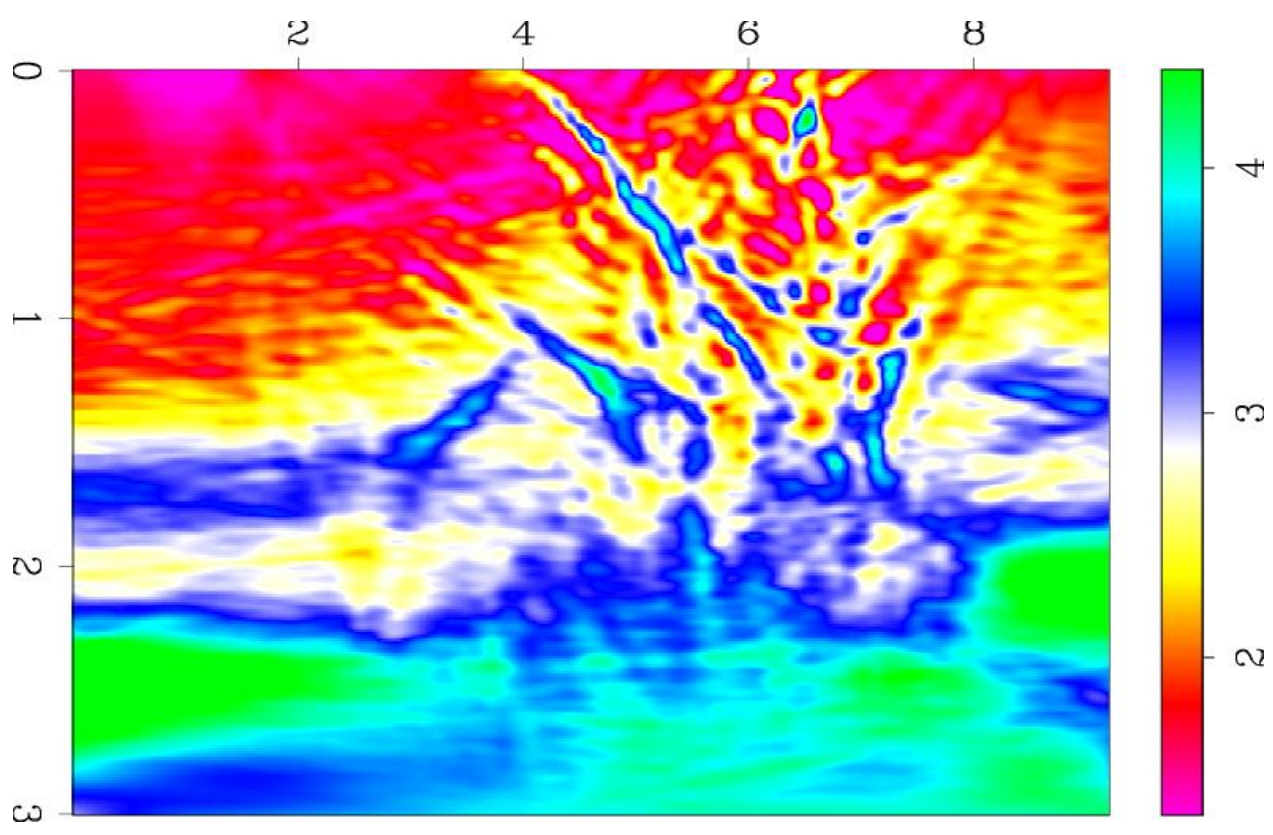

Fig. 21: Velocity output of DSVA to rough Marmousi data at 82th iteration. 


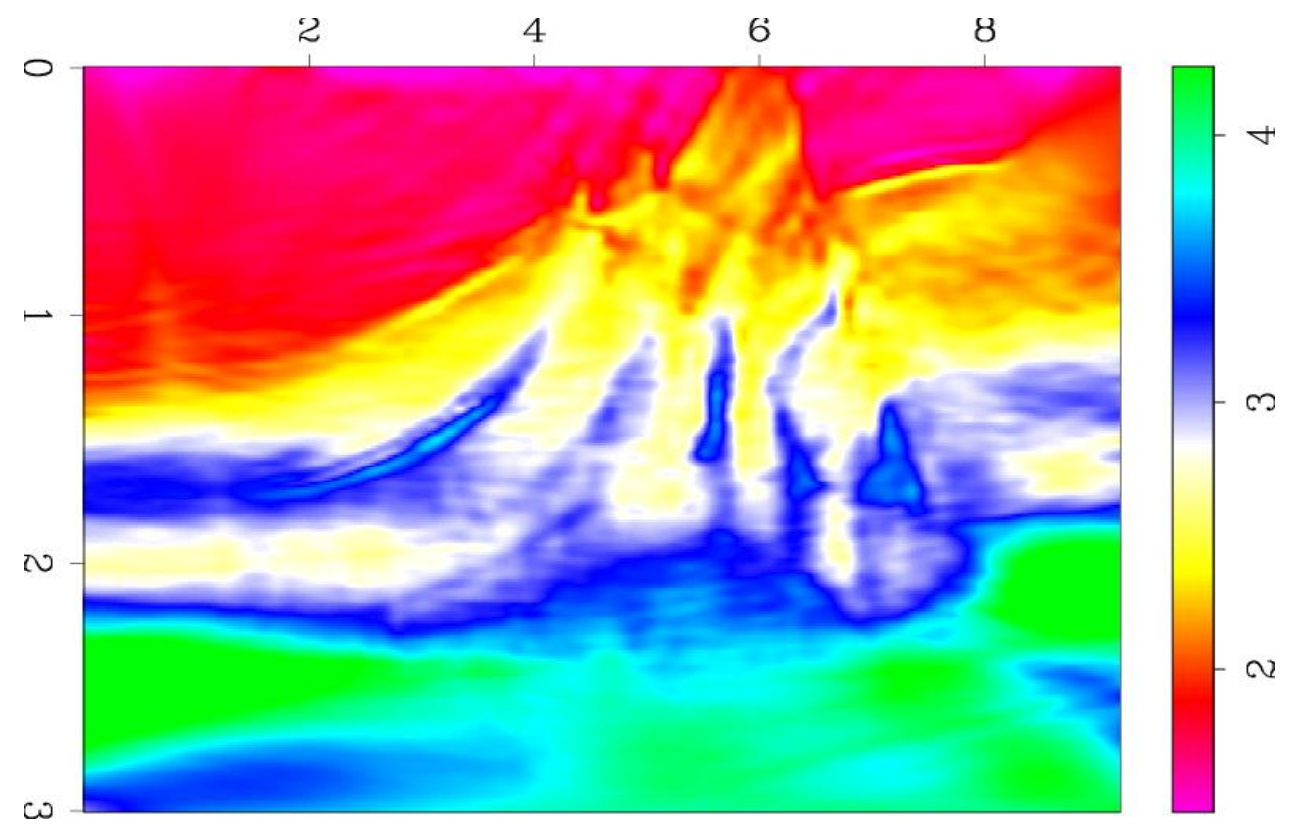

Fig. 22: Velocity output of modified DSVA at 49th iteration.

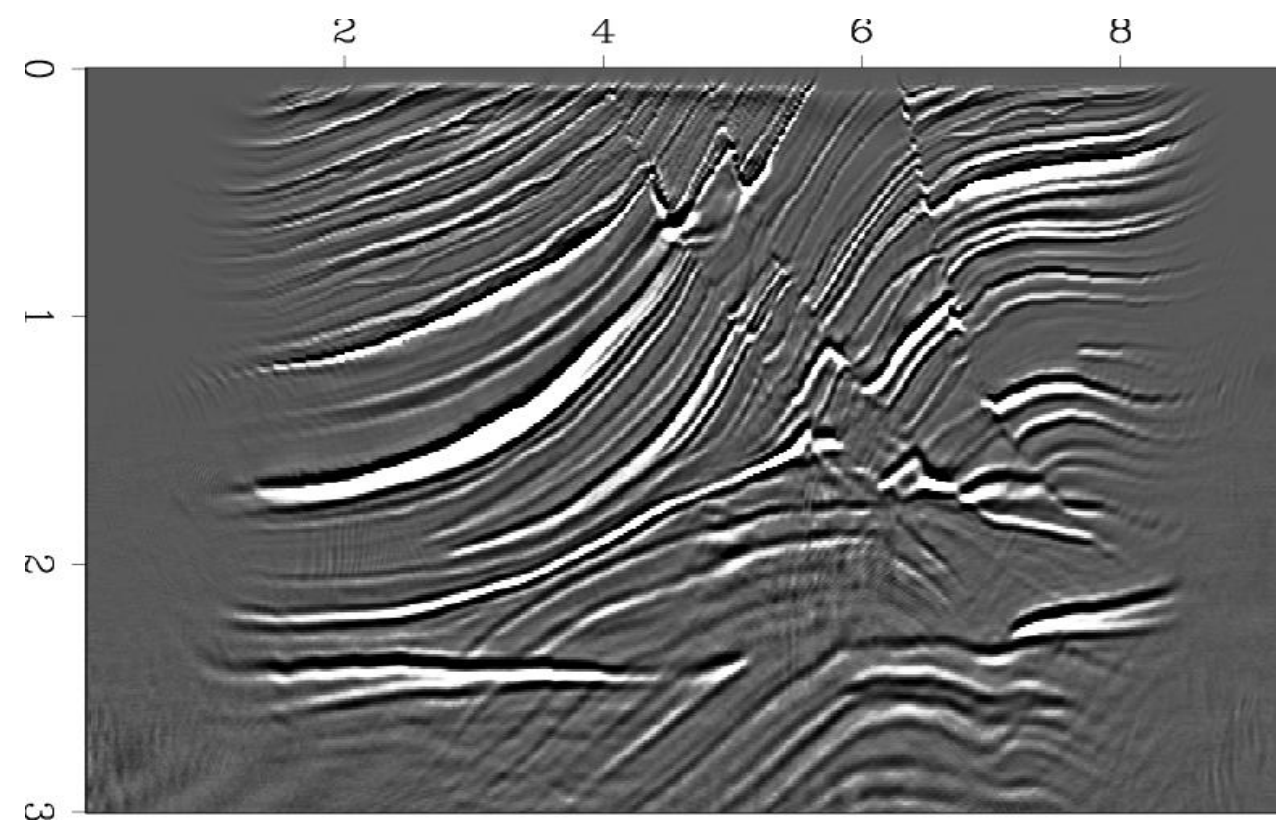

Fig. 23: Image output of modified DSVA at 49th iteration. 


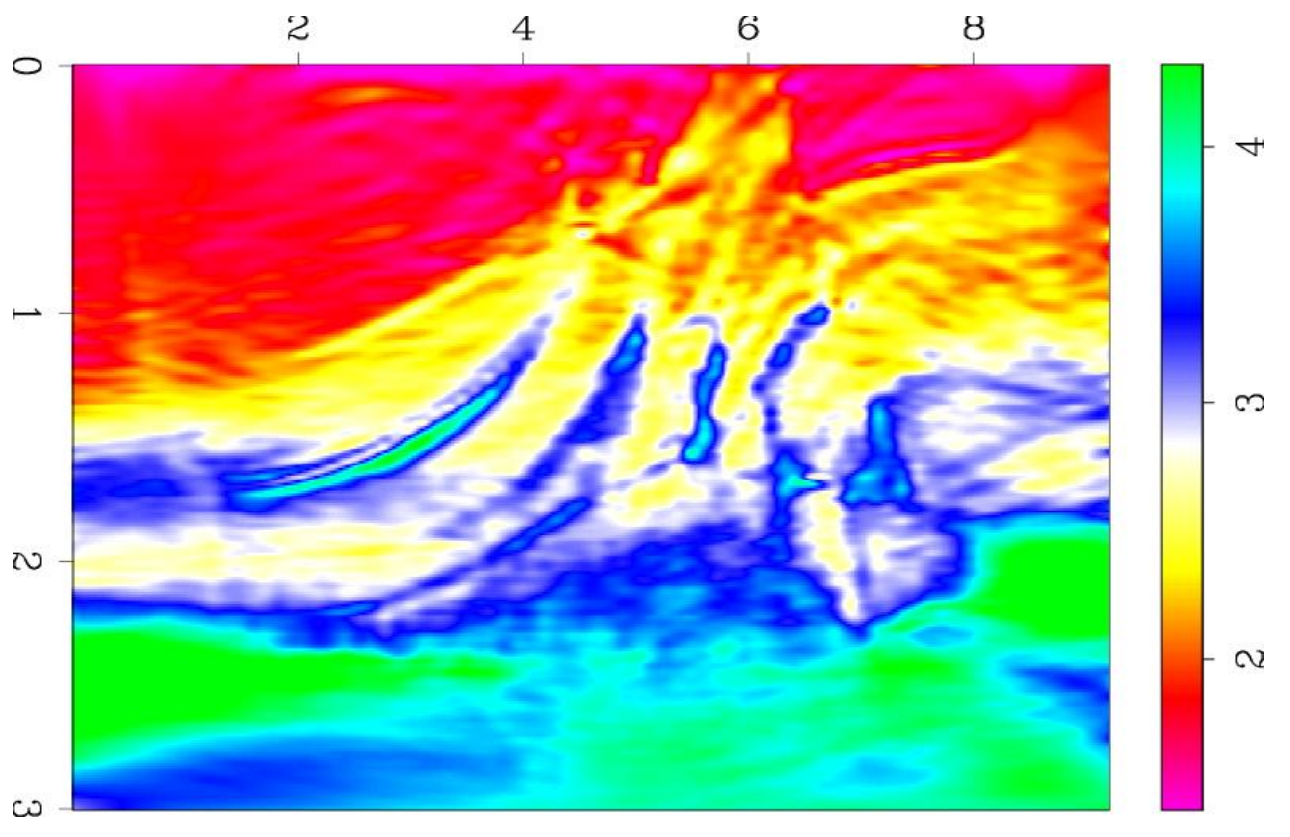

Fig. 24: Velocity output of modified DSVA at 99th iteration.

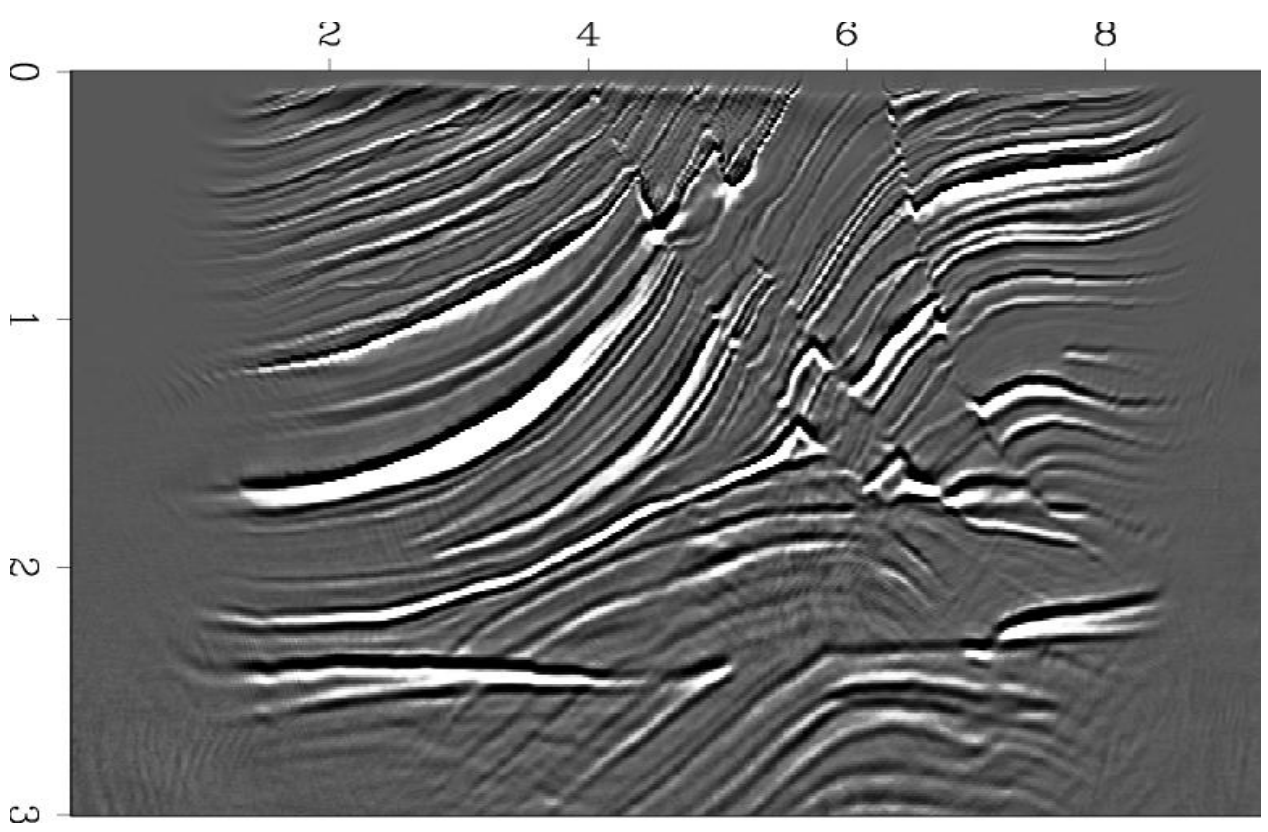

Fig. 25: Image output of modified DSVA at 99th iteration. 


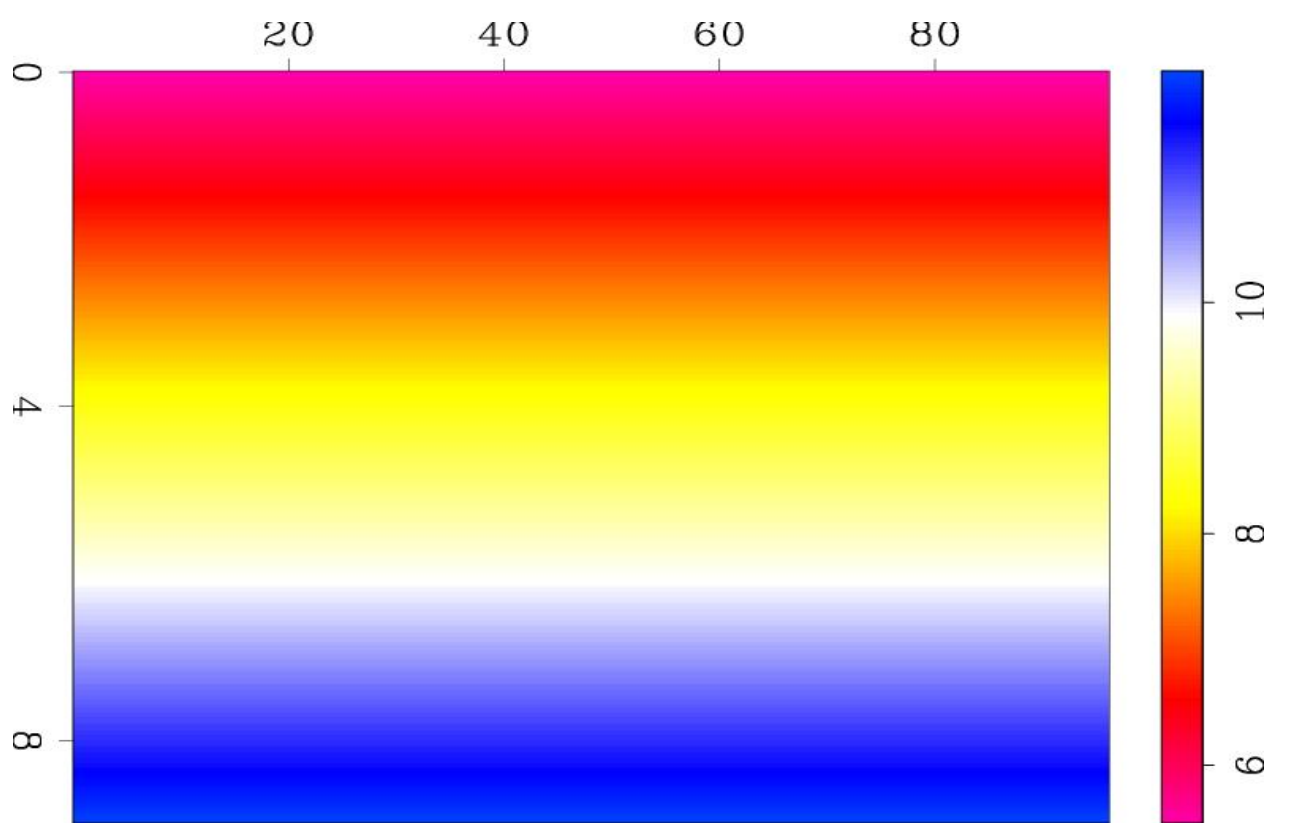

Fig. 26: Initial $v(z)$ velocity model for real data optimization.

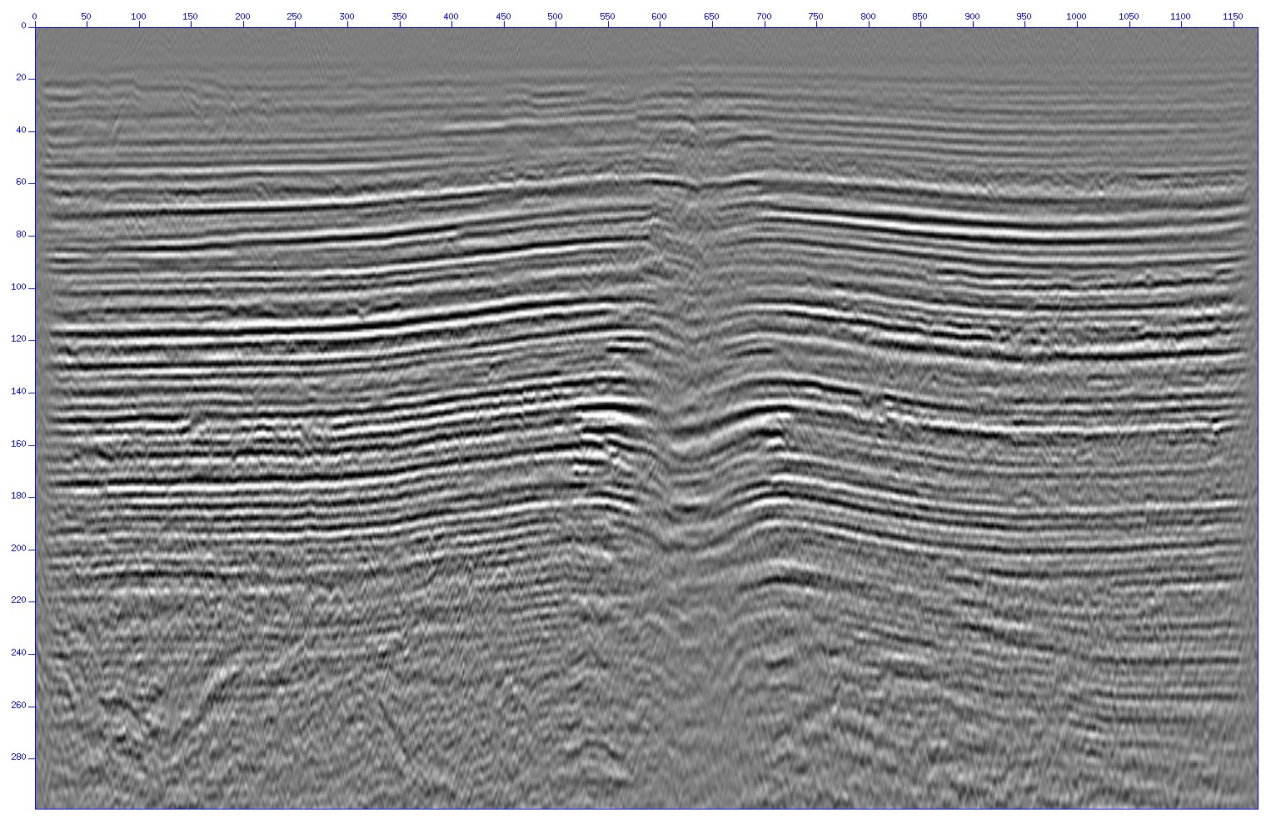

Fig. 27: Initial image by $v(z)$ velocity. 


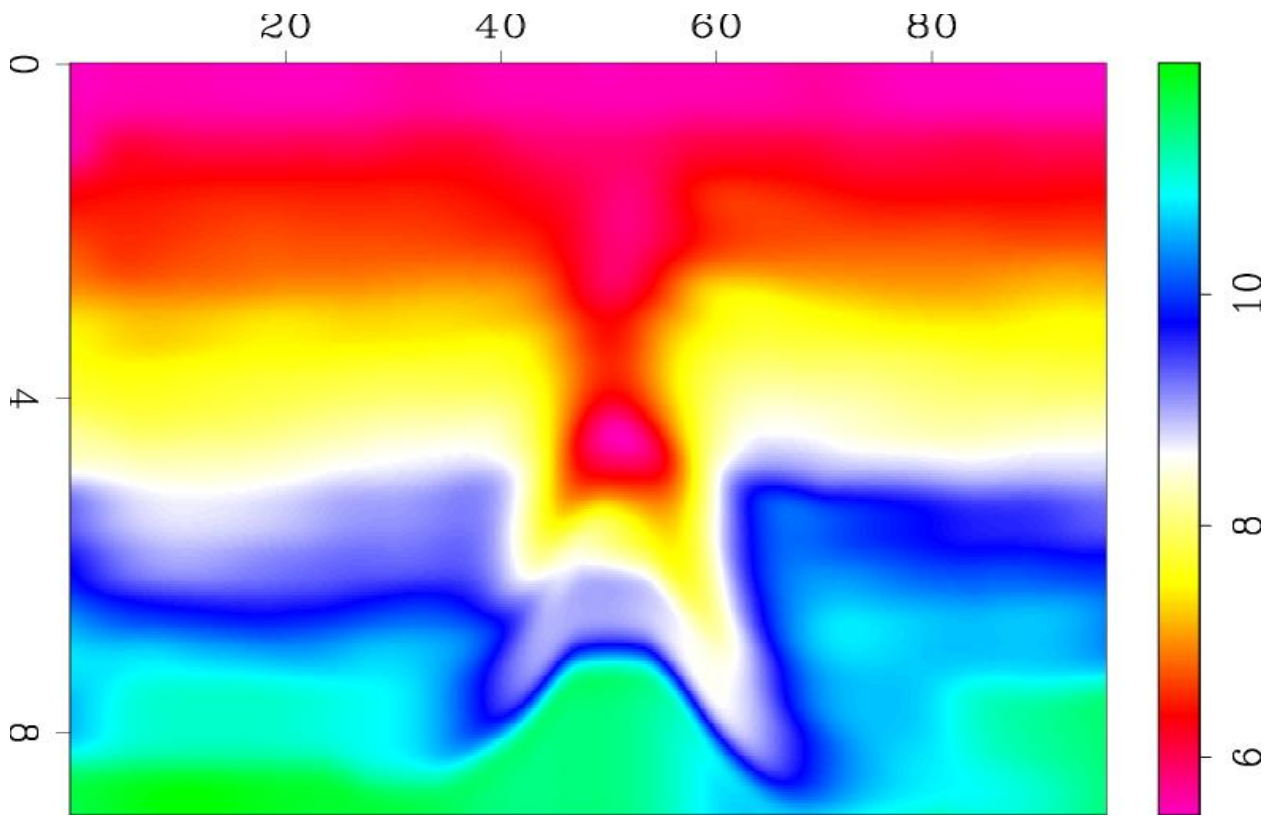

Fig. 28: Optimized velocity by ray tracing tomography.

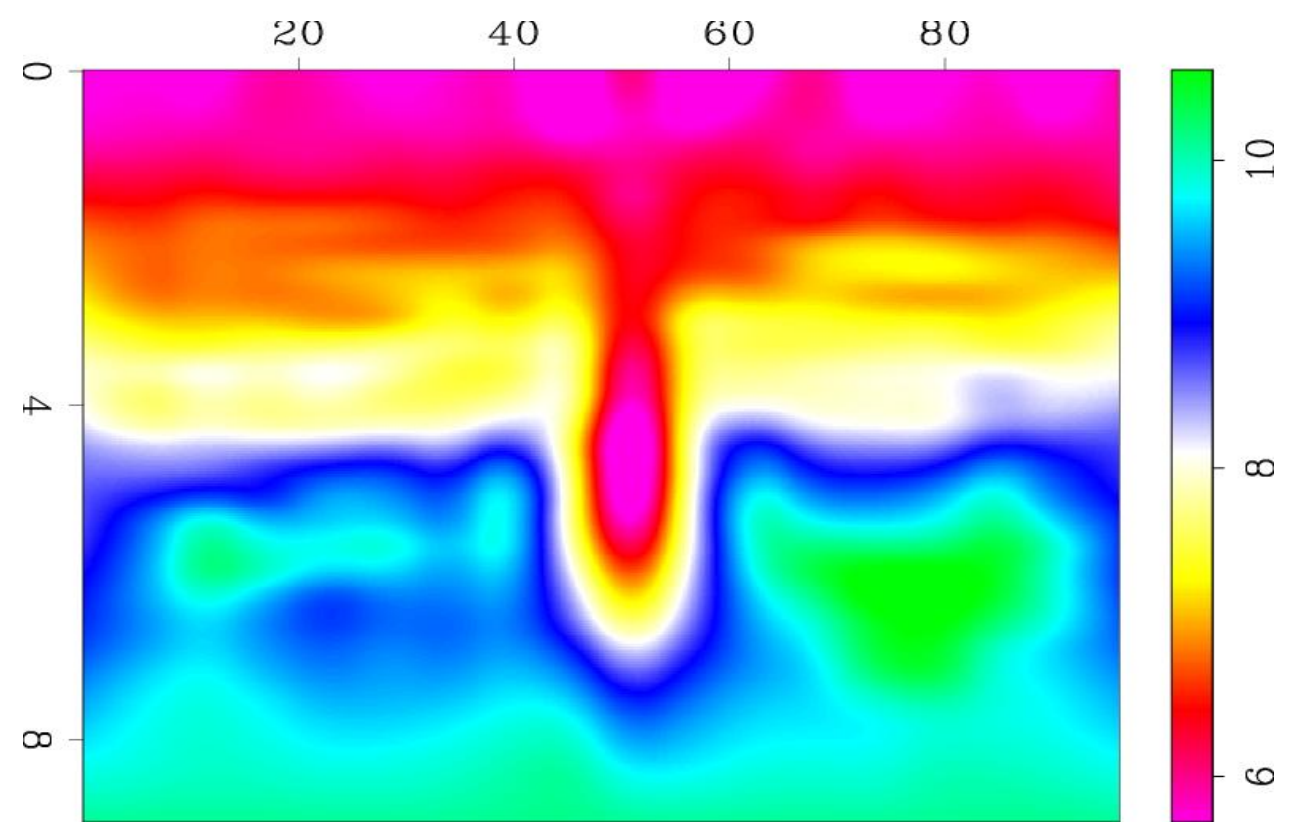

Fig. 29: Optimized velocity by modified DSVA. 


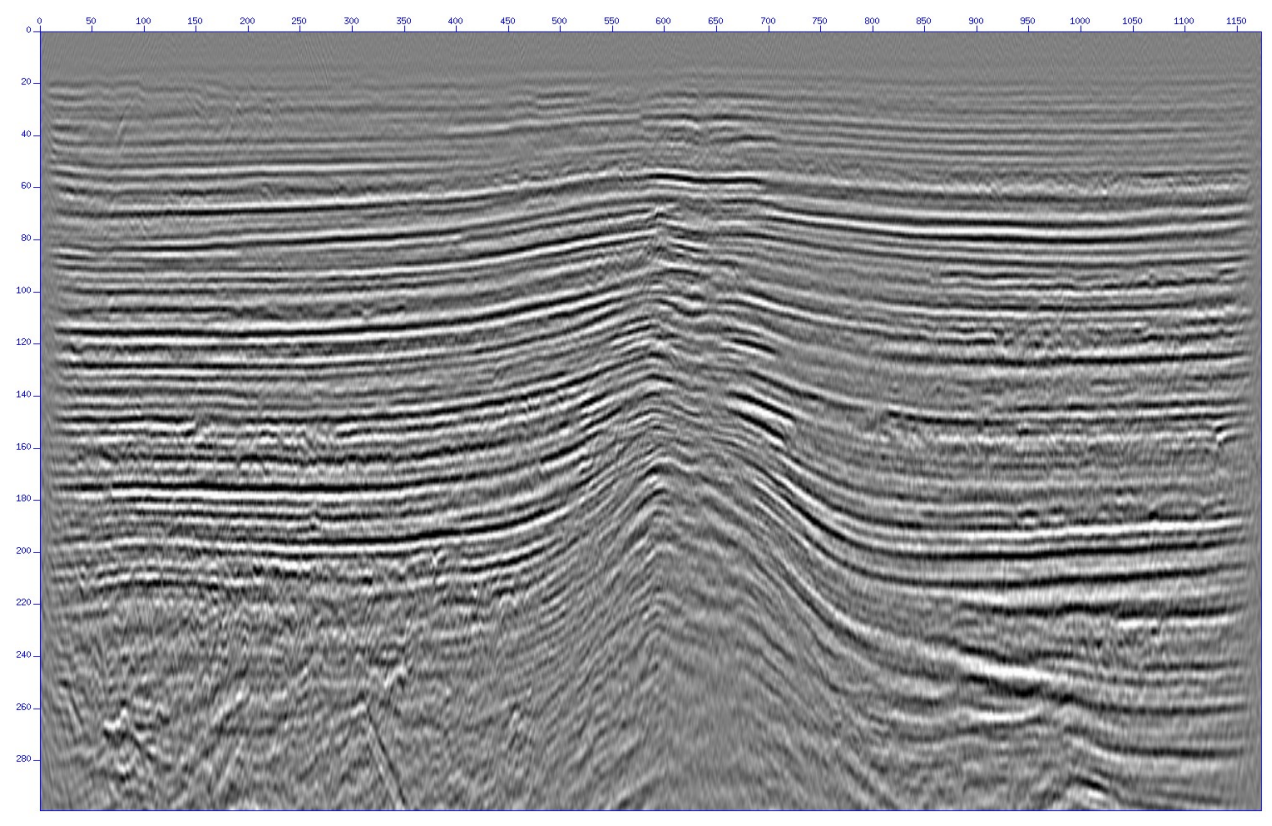

Fig. 30: Image by optimized velocity of ray tracing tomography.

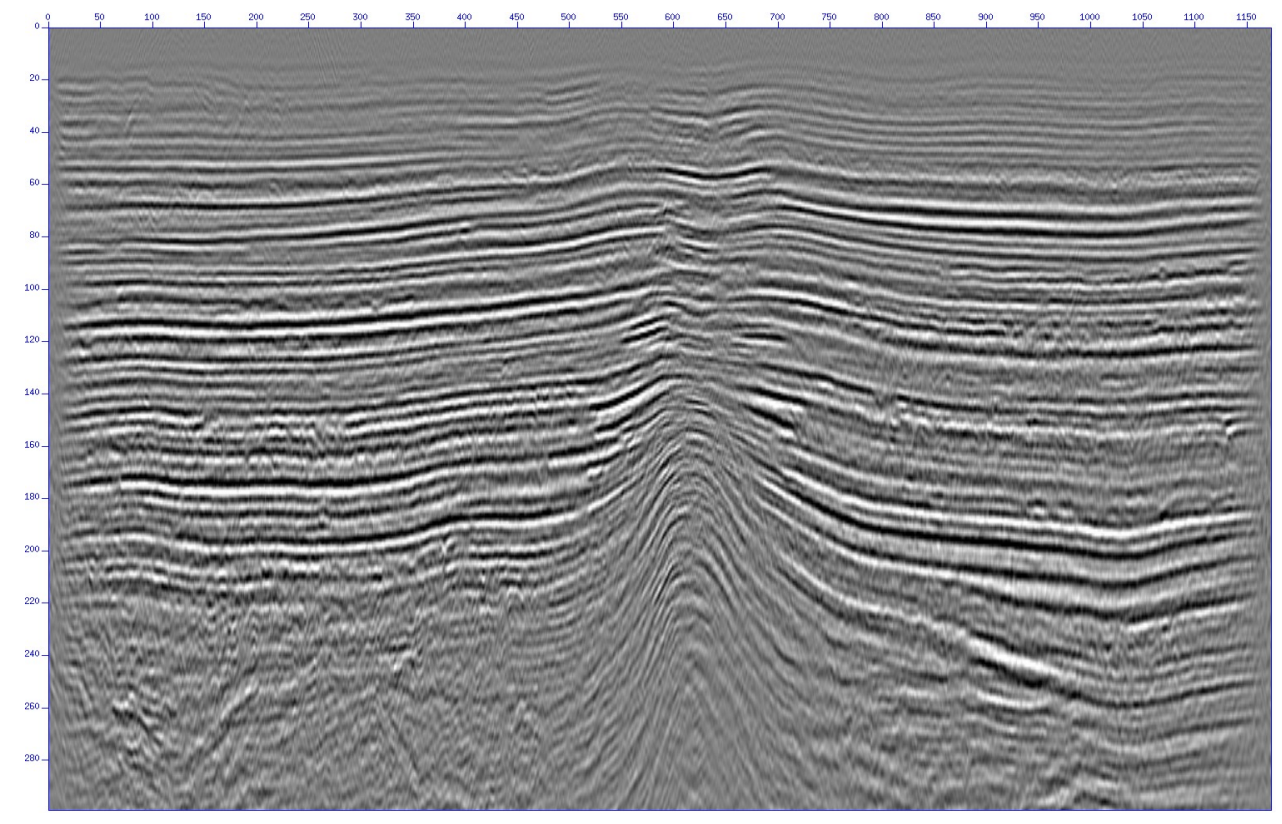

Fig. 31: Image by optimized velocity of modified DSVA. 


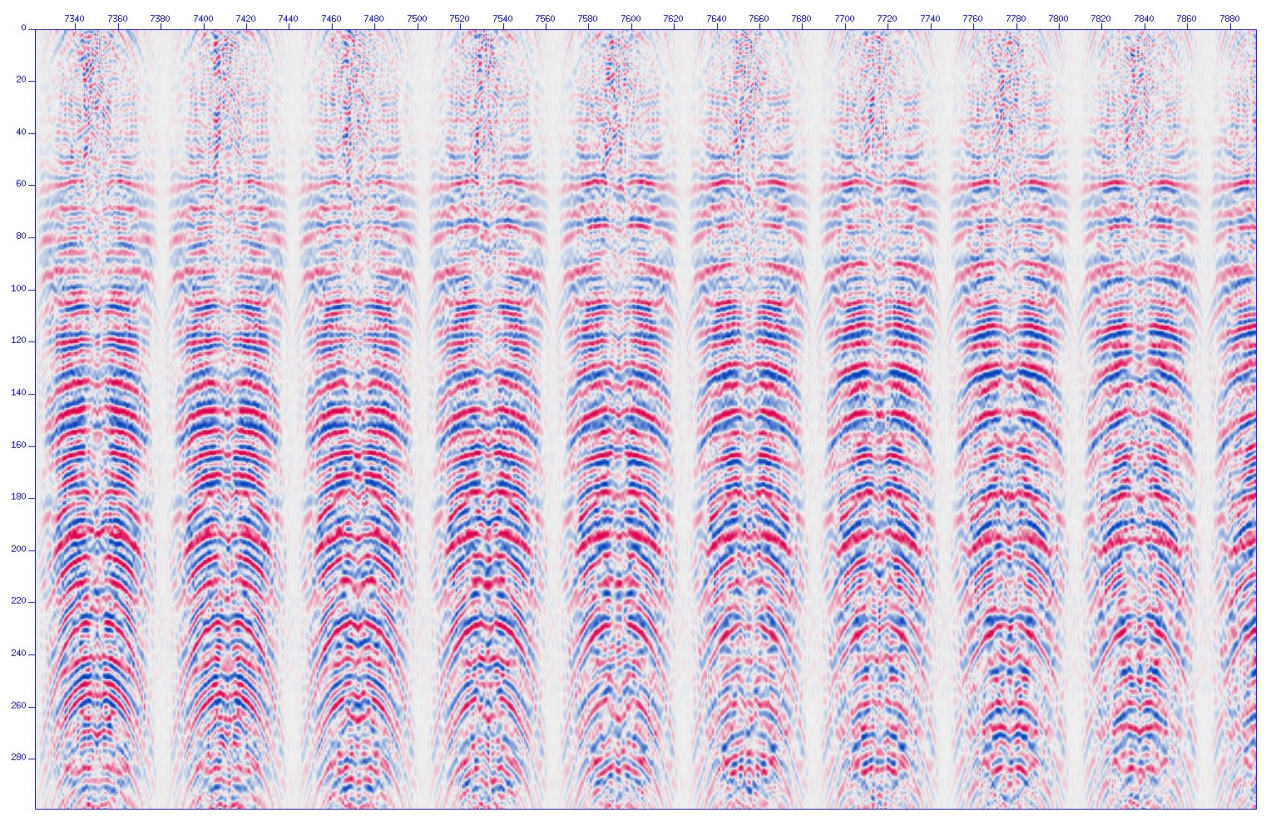

Fig. 32: Initial angle gather by $v(z)$ velocity. 


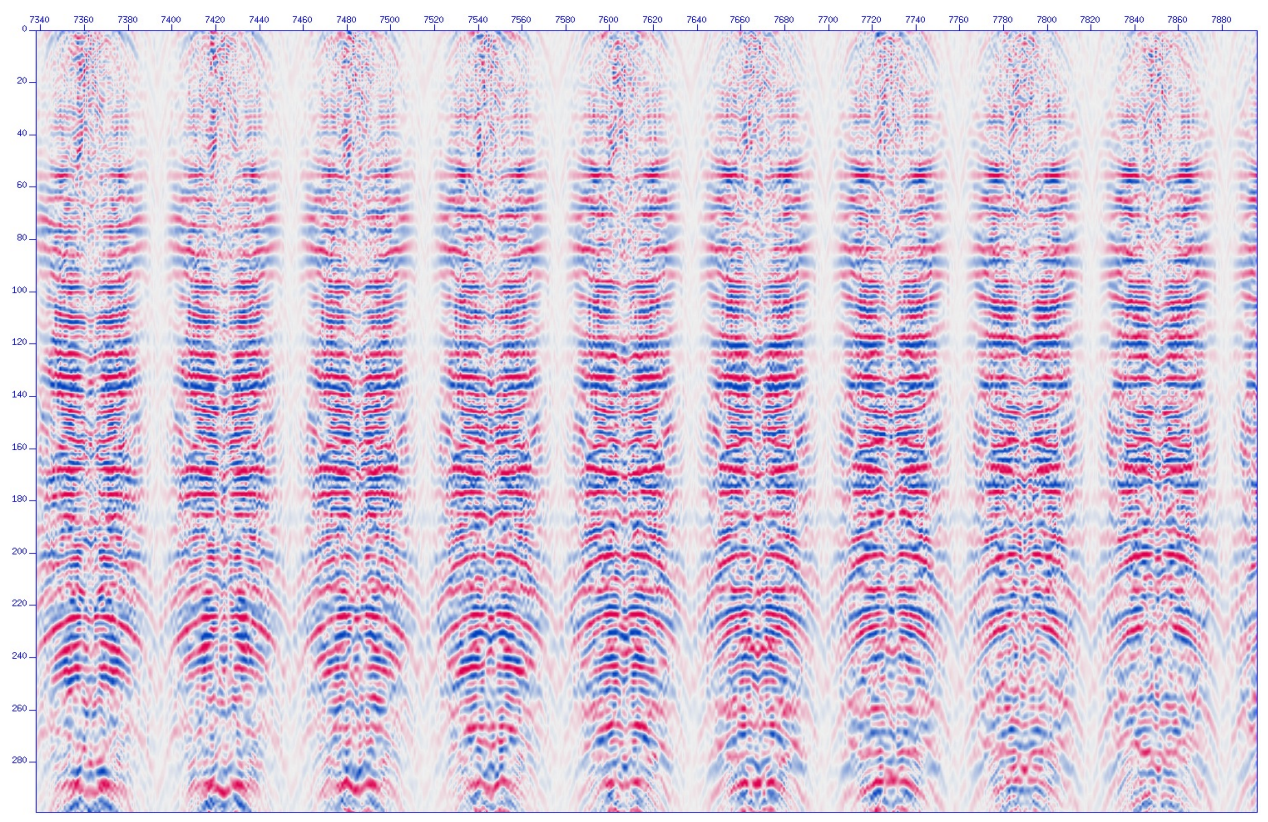

Fig. 33: Angle gather by optimized velocity of ray tracing tomography.

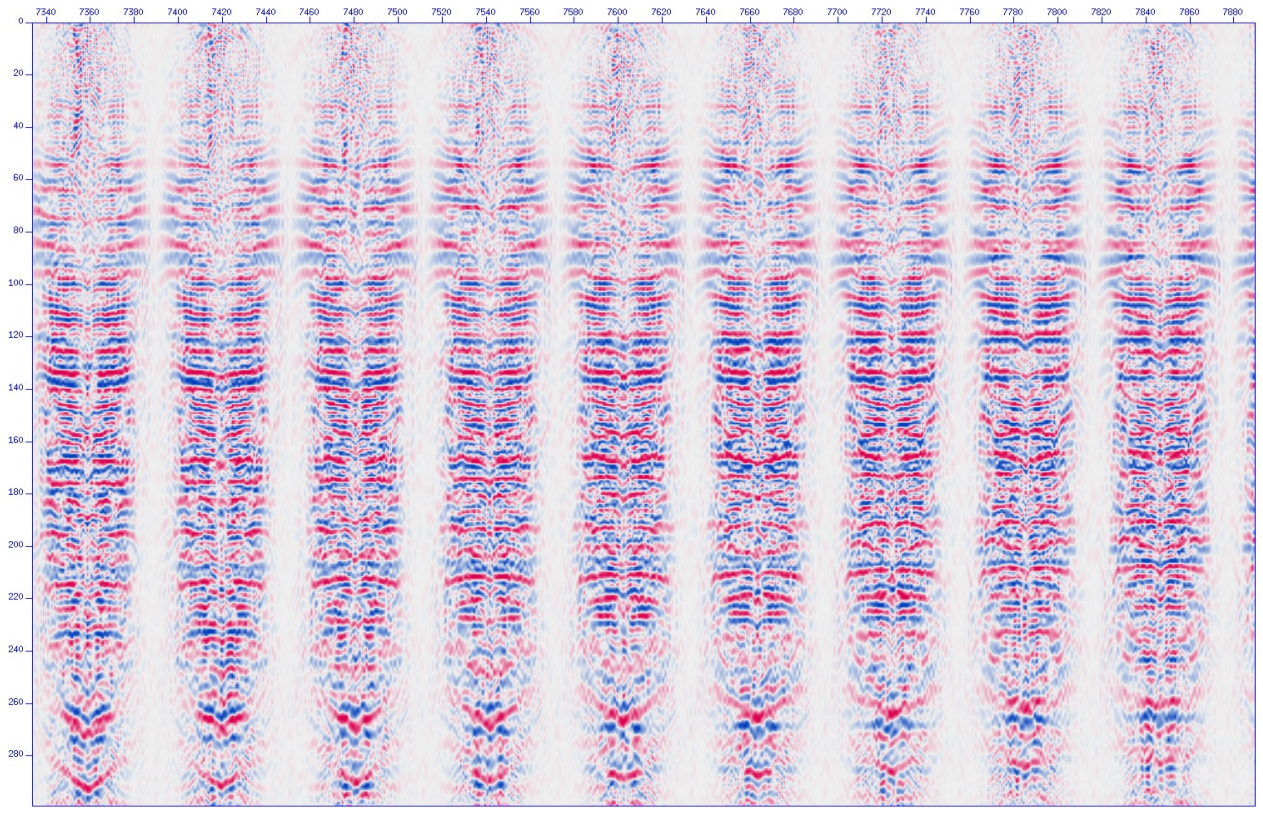

Fig. 34: Angle gather by optmized velocity of DSVA. 